\title{
Vacuole Dynamics in Growth Cones: Correlated EM and Video Observations
}

\author{
M. E. Dailey and P. C. Bridgman \\ Department of Anatomy and Neurobiology, Washington University School of Medicine, St. Louis, Missouri 63110
}

The neuronal growth cone is a major site of surface membrane dynamics associated with uptake and release of materials, motility, and axon extension. Although intracellular membrane organelles are thought to mediate surface membrane addition and retrieval at the growth cone, membrane events are fleeting and therefore difficult to study directly. In an effort to capture transient interactions between intracellular membrane organelles and the plasmalemma at the growth cone, embryonic rat sympathetic neuron cultures were prepared for whole-mount electron microscopy (EM) by rapid freezing and freeze substitution. We identified a set of vacuole-like organelles ( $\geq 150 \mathrm{~nm}$ in diameter) that appeared to interact directly with the plasmalemma. In stereo-pair EM images the bounding membrane of some of these vacuoles had an orifice at sites where the organelle was adjoining the plasmalemma, suggesting that the organelle and surface membranes were confluent. Since this population of organelles could be labeled with cationized ferritin or HRP when added to living cultures just prior to freezing or chemical fixation, they were probably derived from the plasmalemma.

Combined light microscopy and EM of individual growth cones showed that these same vacuoles had a conspicuous reverse shadowcast appearance in differential interference contrast images. Thus, we used real-time video microscopy to follow these organelles in living growth cones. Many of these vacuoles spontaneously appeared, remained visible for several minutes, and then disappeared. Reverse shadowcast vacuoles were formed at various sites throughout the growth cone, including surface membrane ruffles at the leading edge [P (peripheral)-domain] as well as quiescent and retracting regions at the growth cone base [ $C$ (central)domain]. Vacuoles in the P-domain moved centripetally and rarely grew in size. In contrast, those in the $\mathrm{C}$-domain exhibited Brownian-like movements and sometimes appeared to increase in size, raising the possibility that new membrane may be added to these organelles. Vacuoles within both the $\mathbf{P}$ - and $\mathbf{C}$-domains shrank before rapidly disappearing, but rarely vesiculated, suggesting that they had fused with the

\footnotetext{
Received May 28, 1992; revised Feb. 16, 1993; accepted Feb. 22, 1993.

We thank Mr. Grady Phillips for expert technical assistance and Dr. Noreen Reist for comments on an early version of the manuscript. This work was supported by NIH Grants GM08151 and NS26150. We also acknowledge the support of the Integrated Microscopy Resource for Biomedical Research at the University of Wisconsin, Madison.

Correspondence should be addressed to Dr. Paul C. Bridgman, Department of Anatomy and Neurobiology, Box 8108, Washington University School of Medicine, 660 South Euclid Avenue, St. Louis, MO 63110.

a Present address: Department of Molecular and Cellular Physiology, Stanford University School of Medicine, Stanford, CA 94305.

Copyright (C) 1993 Society for Neuroscience 0270-6474/93/133375-19\$05.00/0
}

plasmalemma. The results indicate that vacuoles are a highly dynamic population of organelles that directly communicate with the plasma membrane at the growth cone; they provide a major route of surface membrane uptake and may also play a role in membrane recycling.

[Key words: electron microscopy, video microscopy, pinocytosis, membrane recycling, growth cones, cell culture]

There is a large body of evidence indicating that the growth cone is an active site of uptake of extracellular materials (e.g., Birks et al., 1972; Wessells et al., 1974; Weldon, 1975; Bunge, 1977; Cheng and Reese, 1987). This presumably involves retrieval of surface membrane during endocytosis and pinocytosis, which has long been known to occur at the growth cone (Hughes, 1953; Nakai, 1956). On the other hand, normal axon extension requires a net increase in plasma membrane surface area, and new membrane is thought to be inserted at the growth cone (Bray, 1970, 1973; Pfenninger and Bunge, 1974; Feldman et al., 1981; Pfenninger and Maylie-Pfenninger, 1981; Pfenninger et al., 1992). Therefore, it appears that the growth cone must coordinate membrane addition and retrieval events to subserve net expansion of the surface. One possibility is that retrieved surface membrane is locally recycled and returned to the surface. Indeed, recent studies by Cheng and Reese $(1985,1987)$ have suggested that such a membrane cycle exists in growth cones. These investigators used thin-section electron microscopy (EM) of rapid-frozen specimens to follow the uptake and redistribution of ferritin-labeled surface membrane over time (Cheng and Reese, 1987). Based on this work, they proposed a model of membrane recycling wherein surface membrane is retrieved by vesicles and vacuoles, which are converted to flattened membrane disks, and these disks then add membrane back to the growth cone surface near the base of filopodia. New membrane for growth cone expansion or axon elongation is presumed to be added by fusion of vesicles with vacuole-like precursors of the membrane disks (Cheng and Reese, 1987). Thus, the model suggests that locally recycled membrane and new membrane for addition may converge within large, vacuole-like organelles (see Fig. 7 in Cheng and Reese, 1987). However, it is not known if new membrane is added to vacuoles in this cycle, and there is arguably still no direct evidence for membrane recycling at the growth cone (Gordon-Weeks, 1988).

Although membrane addition and retrieval are thought to be mediated by intracellular membrane organelles, the roles of particular growth cone organelles are not well understood. Nor is it understood how membrane events are coordinated and regulated (spatially and temporally) at the growth cone. This is partly because (1) the membranes of growth cones are extremely sensitive to fixation and therefore prone to disruption when 
prepared for ultrastructural observation by conventional methods (Letourneau, 1985), and (2) membrane events are difficult to observe directly in living growth cones. We have overcome some of these technical problems by using a rapid-frozen wholemount preparation (Bridgman and Dailey, 1989; Dailey and Bridgman, 1991) to identify a set of vacuole-like organelles that interact directly with the plasmalemma of growth cones and that can be labeled with cationized ferritin. We then used highresolution video microscopy to characterize the behavior of reverse shadowcast organelles in live growth cones. These organelles could also be labeled with cationized ferritin or HRP, and they were shown to correspond to the intracellular vacuoles in the EM. The results suggest that vacuole-like organelles play a direct role in retrieving, and probably recycling, growth cone surface membrane. Although they may also mediate addition of some new membrane, the rate of addition is probably not sufficient to fully support growth cone expansion or axon elongation.

\section{Materials and Methods}

\section{Cell culture}

Explant cultures of embryonic rat superior cervical ganglia (SCG) were prepared as described previously (Bridgman and Dailey, 1989; Dailey and Bridgman, 1991). Briefly, pregnant Sprague-Dawley rats (Sasco, Inc., Omaha, NE) were anesthetized with ether or sodium pentobarbital (150 mg/animal) on day 20 or 21 of gestation and the fetuses were removed. Under sterile conditions, SCG were dissected free from surrounding musculature and transferred to a culture dish containing L15 media. SCG were decapsulated, cut into 8-12 pieces each, and explanted onto formvar-coated gold (200 mesh hex or finder) EM grids or acidcleaned glass coverslips, both previously coated with laminin (15-20 $\mu \mathrm{g} / \mathrm{ml}$ in $0.05 \mathrm{M}$ carbonate buffer, $12 \mathrm{hr}$ ). The culture medium was an Eagle's minimum essential medium (GIBCO) base containing 10\% horse serum, $2 \%$ chick embryo extract, $0.6 \%$ glucose, and nerve growth factor $(50 \mathrm{ng} / \mathrm{ml}$; gift of E. Johnson, Washington Univ.). Cultures were grown overnight in a humidified environment with $5 \% \mathrm{CO}_{2}$ at $35^{\circ} \mathrm{C}$, and growth cones were examined on the following day.

\section{Electron microscopy}

Rapid freezing and freeze substitution. Neuronal cultures were preserved by rapid freezing and freeze substitution because (1) primlary fixation in glutaraldehyde can disrupt the plasma membrane and artificially induce formation of intracellular organelles (Rees and Reese, 1981), and (2) rapid freezing "fixes" cells at a faster rate so that transient membrane events may be captured (e.g., Heuser et al., 1979; Knoll et al., 1987; Sitte et al., 1987). Procedures for rapid freezing and freeze substitution of whole-mount growth cones were described previously (Bridgman and Dailey, 1989; Dailey and Bridgman, 1991). Briefly, explants on EM grids were rapidly frozen by plunging into a stirred mixture of propane/ ethane $(2: 1)$ cooled by liquid nitrogen. Specimens were freeze substituted in absolute acetone (Aldrich, Milwaukee, WI) or $90 \%$ methanol containing the following sequence of fixatives: 10\% acrolein (overnight, $\left.-80^{\circ} \mathrm{C}\right), 0.4 \%$ osmium $\left(2-3 \mathrm{hr},-20^{\circ} \mathrm{C}\right)$, and $10 \%$ glutaraldehyde (overnight, $4^{\circ} \mathrm{C}$ ). Staining was with $0.5 \%$ uranyl acetate and $0.5 \%$ hafnium chloride. Following freeze substitution, specimens were critical point dried and coated with a thin layer of carbon. Freeze substitution in either acetone or methanol yielded similar results, although intracellular membrane organelles were more clearly visible in methanol-substituted specimens.

Chemical fixation. To allow light microscopy (LM) and electron microscopy of the same growth cone, some cultures grown on EM finder grids were fixed with a warm solution of $0.25 \%$ glutaraldehyde in 0.1 M cacodylate buffer, $\mathrm{pH} 7.4\left(+5 \mathrm{~mm} \mathrm{CaCl}_{2}\right)$, for $10 \mathrm{~min}$. They were then further fixed with $1 \%$ glutaraldehyde in the same buffer at room temperature, followed by $0.1 \%$ osmium tetroxide (in buffer without the $\mathrm{CaCl}_{2}$ ) for $20 \mathrm{~min}$ on ice. The cultures were stained with $1 \%$ aqueous uranyl acetate for $10 \mathrm{~min}$ prior to dehydration and critical point drying.

Ferritin labeling of surface membrane. Cationized ferritin was used to mark intracellular membrane derived from the plasmalemmal surface. SCG explant cultures grown on EM grids (normal or finder type) were labeled with cationized ferritin (Sigma, cat. \#F7879) for 1-15 min in the following manner (after Cheng and Reese, 1987). The normal culture medium (buffered with bicarbonate) was replaced with warm L15 media (buffered with HEPES), followed by incubation (at $35^{\circ} \mathrm{C}$ ) in $\mathrm{L} 15$ containing $4 \mathrm{mg} / \mathrm{ml}$ ferritin for the indicated duration (see Results). At the appropriate time, grids were rinsed once with L15, rapidly frozen, and then freeze substituted in methanol, or chemically fixed, as described above.

Labeling with horseradish peroxidase. The normal culture medium was replaced with medium containing $10 \mathrm{mg} / \mathrm{ml}$ horseradish peroxidase (HRP; Sigma). After $15 \mathrm{~min}$ at $35^{\circ} \mathrm{C}$, the cultures were washed with three changes of normal medium over a $3 \mathrm{~min}$ period and then chemically fixed with $0.25 \%$ glutaraldehyde as described above. To visualize the HRP, a reaction was carried out using the Hanker-Yates protocol (Hanker et al., 1977). The reaction time was $10 \mathrm{~min}$. Following completion of the HRP reaction, the cultures were further fixed with glutaraldehyde and osmium using the same procedure as described above.

Electron microscopy. Whole-mount growth cones were viewed at 120 $\mathrm{keV}$ using a JEOL 1200 electron microscope or at $1000 \mathrm{keV}$ using an A.E.I. EM-7 high-voltage electron microscope (University of Wisconsin, Madison).

\section{Video microscopy}

Differential interference contrast video microscopy. Growth cones were observed using high-resolution, video-enhanced contrast-differential interference contrast (DIC) microscopy (Allen et al., 1981; Inoue, 1981), as described previously (Dailey and Bridgman, 1989). An inverted microscope (Zeiss IM-35) was used with either a $63 \times(1.4 \mathrm{NA})$ or a $40 \times$ (1.3 NA) Zeiss oil-immersion objective. The latter lens was used only when additional working distance was required to visualize cells grown on EM finder grids. The light source was a $100 \mathrm{~W}$ mercury arc lamp adjusted for critical illumination. The specimens were viewed with a Newvicon camera (Ikegami, Tokyo) coupled with a $12.5 \times, 20 \times$, or $25 \times$ projection eyepiece. To reduce the heat load on the specimens, heat reflection and heat adsorption filters were placed in series in the path of illumination. The light was made monochromatic $(546 \mathrm{~nm})$ using an interference filter. Real-time background subtraction was performed with an image processor (Hughes, model 794) to obtain a mottlefree image. Images were stored on magnetic tape (Sony U-matic videocassette recorder model VO-5800) or on optical memory disk (Panasonic recorder model TQ-2028F).

Living cultures were mounted in a closed chamber and secured to a metal holder with adhesive tape. The entire assembly was placed on a heated $\left(35-37^{\circ} \mathrm{C}\right)$ copper stage of the inverted microscope, and the chamber temperature was continuously monitored using a miniature thermocouple attached to the coverslip with thermoconductive paste (Omega Engineering, Stamford, CT). Fixed cultures on EM grids were mounted in a closed chamber similar to the one used for living cultures except that the chamber depth was greater. The fixed cells were imaged at room temperature.

\section{Results}

When grown on a laminin-containing substratum, neurites of rat sympathetic neurons typically extend at rates between 10 and $50 \mu \mathrm{m} / \mathrm{hr}$ (e.g., Kleitman and Johnson, 1989). Although the growth cones at the tips of these neurites exhibit morphological variability, most of them are characterized by two distinct cytoplasmic domains: a microtubule-rich central (C-) domain and an actin-rich peripheral (P-) domain (Bridgman et al., 1986; Bridgman, 1992). Growth cones contain a variety of intracellular membrane organelles, most of which are restricted to the C-domain, but here we describe observations on vacuole-like organelles that were found in both the C-and P-domains.

\section{Whole-mount EM of rapid-frozen growth cones}

Most of the rapid-frozen whole-mount growth cones we examined contained some large ( $\geq 150 \mathrm{~nm}$ ), clear vacuole-like organelles. These organelles had round, oval, or oblong shapes, and they ranged in size from $150 \mathrm{~nm}$ to about $2 \mu \mathrm{m}(687 \pm 434$ $\mathrm{nm}$, mean $\pm \mathrm{SD}$ ) in the longest dimension. Virtually all of the 

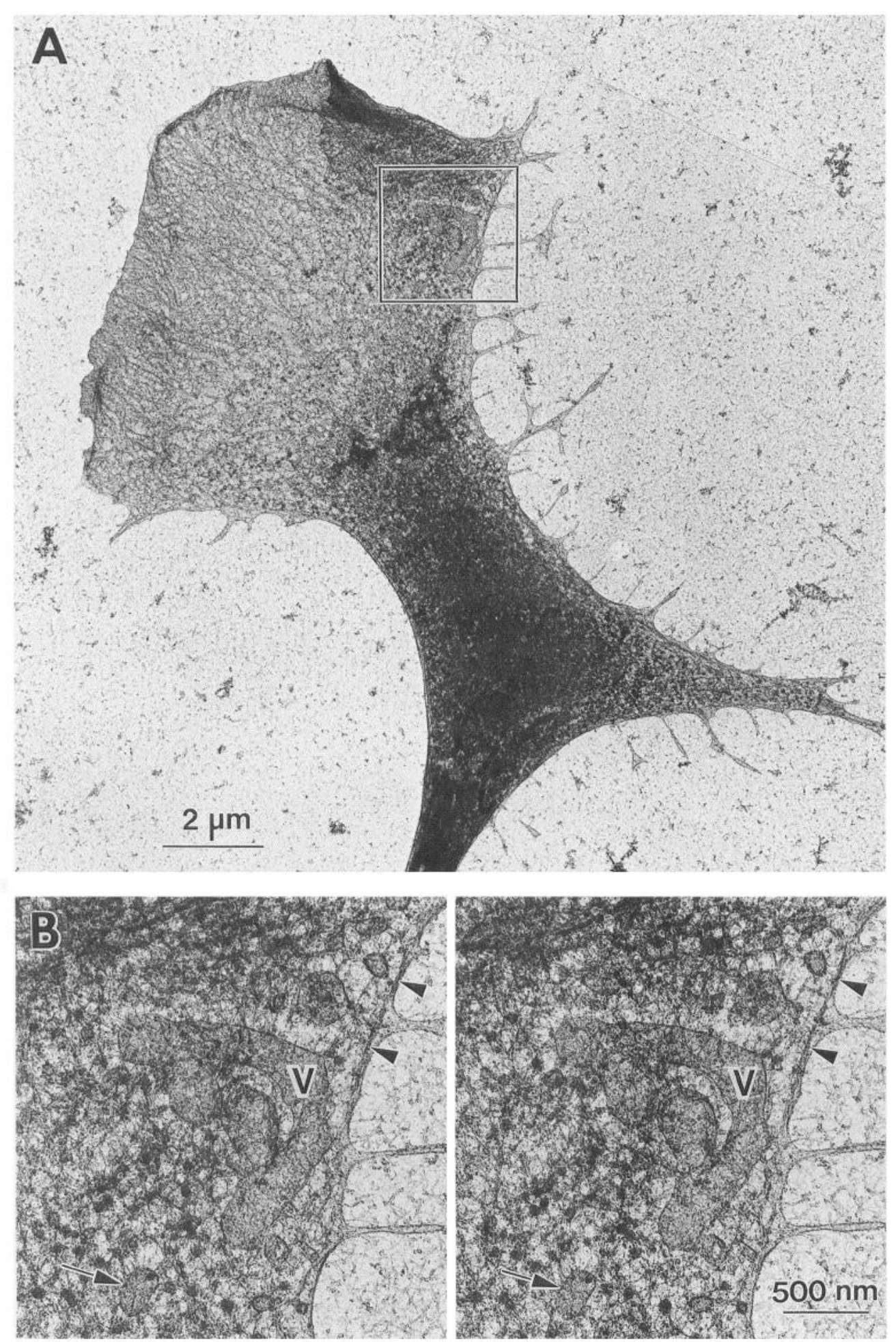

Figure 1. Growth cones of rat sympathetic neurons often contained rather large vacuole-like organelles. $A$, A small cluster of vacuoles (box) is located adjacent to a scalloped-shaped region along the lateral margin of the growth cone. Contrast the appearance of this region with the more smoothly contoured margin at a presumptive site of protrusion at the left edge of the growth cone. $B$, Stereo-pair micrographs of boxed region in $A$ showing both large $(V)$ and small (arrow) vacuoles. Note the filaments (arrowheads) oriented parallel with the margin, which indicate a stable or retracting region of the growth cone (cf. Tosney and Wessells, 1983). growth cones contained at least one of these organelles, while some growth cones had as many as 15 . These organelles were most often located in the C-domain near regions that were expected to be stable or retracting, but not protruding, at the time of freezing (Fig. 1).

We examined over a thousand growth cones for evidence of direct physical interaction between vacuoles and the surface membrane. Careful study of high-magnification stereo-pair images suggested that the bounding membrane of a few (about 20) vacuole-like organelles was confluent with the plasmalemma. These organelles had a single circular or oval orifice $(175 \pm 56$ $\mathrm{nm}$ in diameter) in the bounding membrane, and this orifice was consistently located within the plane of the plasmalemma. The walls of the vacuole-like organelle and the plasmalemma appeared to emanate from the lip of the orifice. In other words, there appeared to be a hole in the plasma membrane precisely where there was a hole in the adjacent vacuole membrane (Fig. 2 ). This appearance is what one would expect if an intracellular 
Figure 2. Some vacuoles appeared to be confluent with the plasmalemma of rapid-frozen growth cones. $A$, Stereopair micrographs of a cluster of vacuoles near the margin of a growth cone. Several of the vacuoles had an orifice (single arrowheads) that was in the plane of the plasmalemma when viewed stereoscopically. Note that different organelles were confluent with either the upper (arrowheads) or lower (arrowhead with $\left.{ }^{*}\right)$ plasmalemmal surfaces. The alignment of filaments (double arrowheads) parallel with the concave margin suggests that this region was not protruding at the time of freezing. $B$, Highmagnification stereo-pair micrographs of a different growth cone demonstrating an oval orifice (arrowheads) in the membrane of the sac-like organelle. The orifice is in the plane of the upper plasmalemmal surface, and the body of the organelle appears to originate from the lip of the orifice. Note that the area circumscribed by the orifice appears lighter (less heavily stained) than surrounding regions, which we interpret to be due to the absence of membrane.
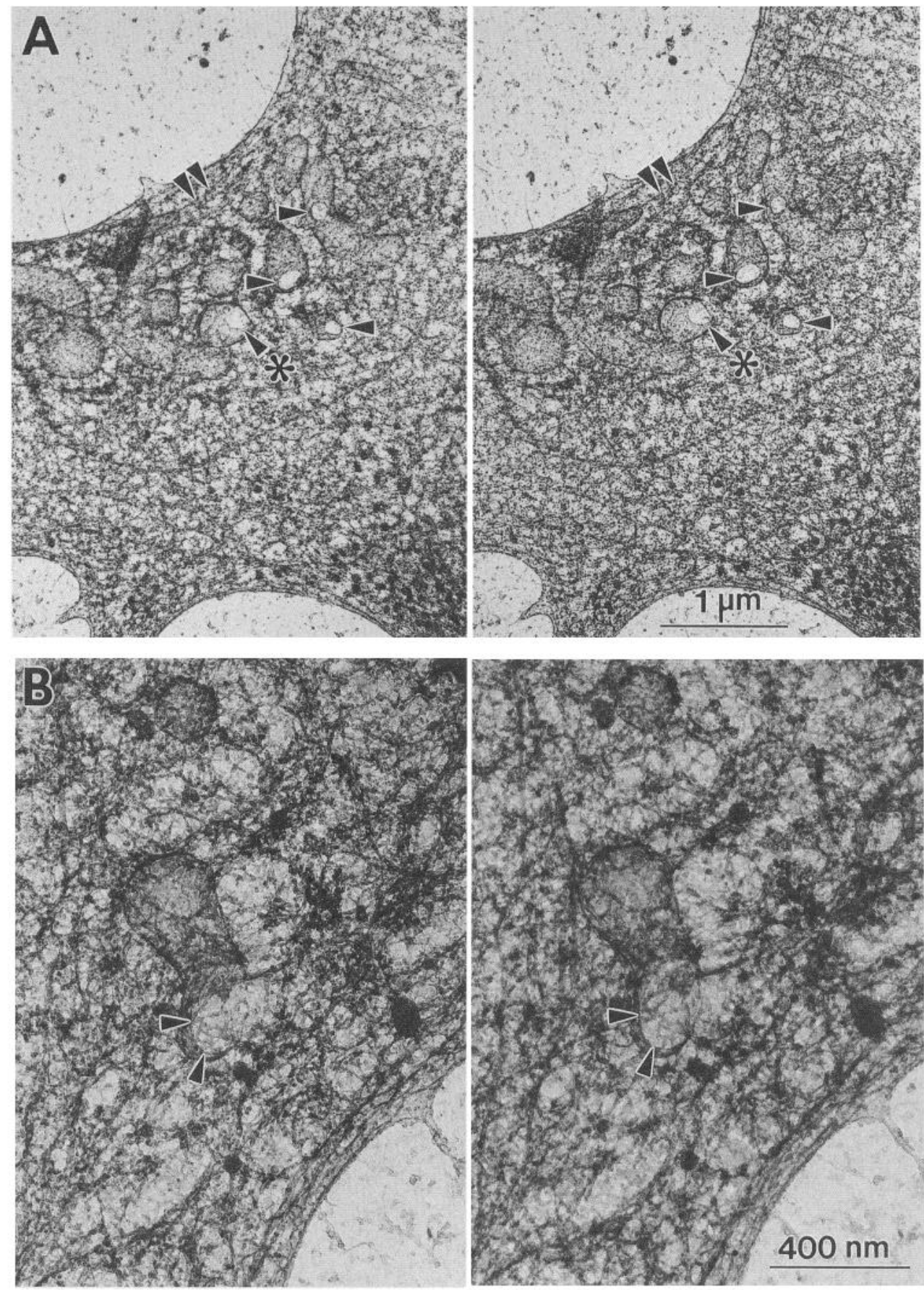

organelle had fused with the plasmalemma, or if the surface membrane was invaginated at that location. Interestingly, this organelle-surface membrane interaction was observed at both the free (top) and substratum-apposed (bottom) membrane surfaces of growth cones (Fig. $2 A$ ), suggesting that this phenomenon was not induced by an unusual interaction (or lack of interaction) with the culture substratum.

Vacuoles with an orifice were found at the base of growth cones near regions that were expected to be retracting or stabilized (Fig. 2). They were also found near the center of the growth cone $\mathrm{C}$-domain, several microns away from the margins (Fig. 3), indicating that they were not associated exclusively with retracting margins. Although vacuole-like organelles were oc- casionally located near the leading edge of the growth cone when membrane ruffling was apparent, none of the vacuoles within the ruffles had an orifice or appeared to be confluent with the plasmalemma (Fig. 4).

Labeling of membrane with cationized ferritin. The confluence of the vacuole and plasma membranes could indicate either membrane addition to, or retrieval from, the growth cone surface. To determine if surface membrane was being retrieved by any of the vacuoles, we incubated cultures in cationized ferritin for a short time (1-15 min) just prior to freezing (see Materials and Methods). This treatment ought to label the plasma membrane as well as any intracellular membranes that were derived from the plasma membrane in the time period following the 

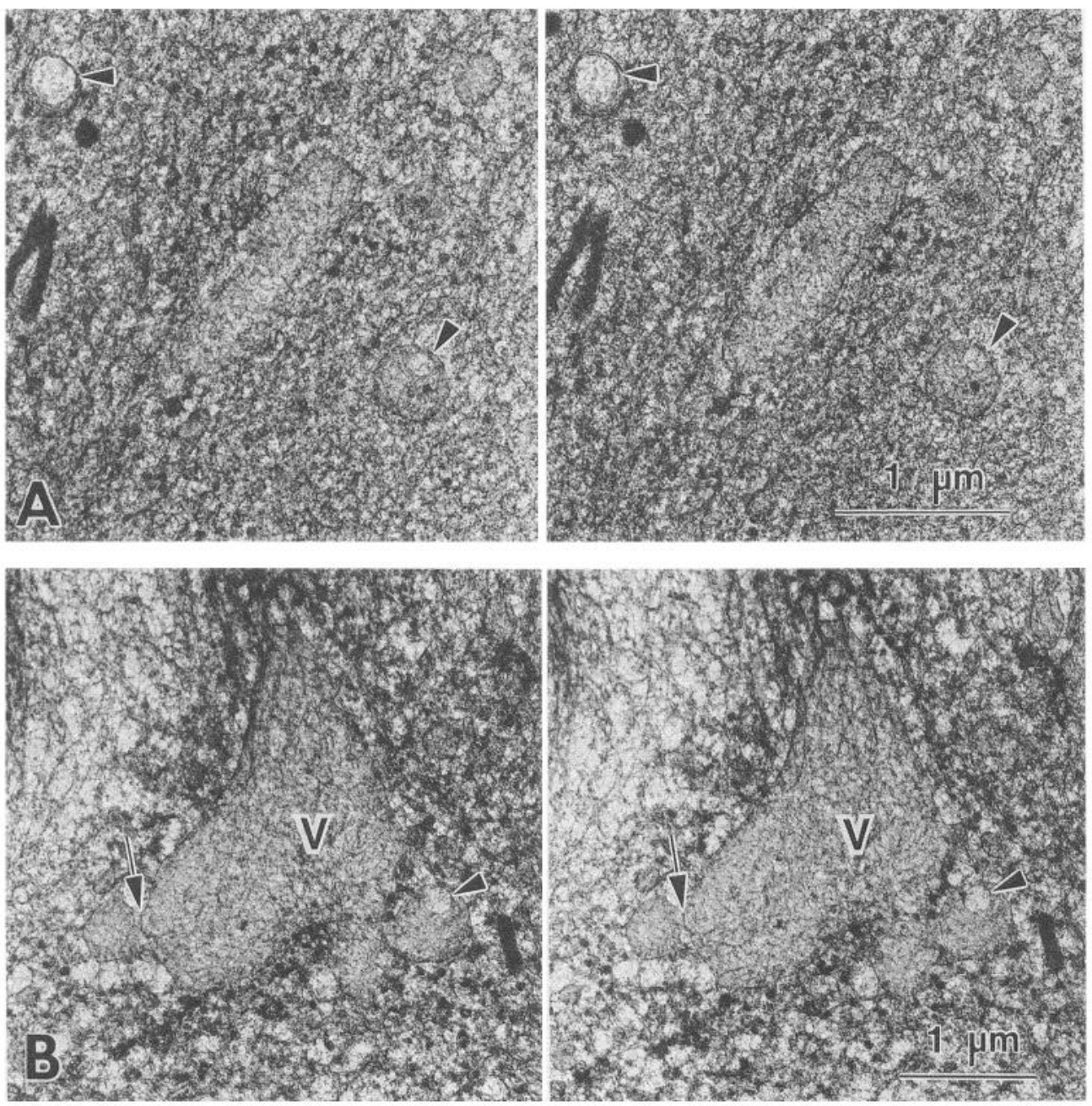

Figure 3. Some vacuoles near the center of the growth cone had an orifice (arrowheads). A, The organelle at the upper left has a large orifice and opens up to the free (upper) plasmalemmal surface, while another organelle at lower right interacts with the lower (substratum-associated) surface membrane. $B$, A large vacuole $(V)$ in the $C$-domain interacts with other smaller vacuoles, one of which has an orifice (arrowhead). A contact site (arrow) between the large vacuole and a smaller organelle may indicate fusion or budding. ferritin application. When the labeled cultures were frozen, processed, and examined in the EM, we observed that ferritin particles and clumps covered the surfaces of growth cones and the formvar substratum. In addition, some of the intracellular vacuoles appeared much more dense than normal, suggesting that they contained the ferritin and were therefore derived from the plasmalemma (Fig. 5). The labeled vacuoles had a similar overall appearance, distribution, and size $(470 \pm 160 \mathrm{~nm})$ as the clear vacuoles described above. However, we did not find any ferritin-labeled vacuoles that had an orifice in the bounding membrane or that were unequivocally confluent with the plasma membrane.

To better determine the significance of ferritin-labeled vacuoles in the overall membrane economy of growth cones, we attempted to analyze these organelles in a quantitative manner. There was some difficulty initially with this analysis because of the heterogeneity of the vacuole types seen in growth cones. Vacuoles vary in electron density even within individual unlabeled growth cones, and differences in uranyl acetate staining from sample to sample can give rise to further variations. We therefore analyzed all vacuoles in a number of both control and ferritin-labeled growth cones and placed them into one of four categories (Table 1). Type III and IV vacuoles were never seen in control growth cones and therefore probably represent ferritin-laden vacuoles. Supporting this assumption is the increase in frequency of these vacuole types with increased ferritin incubation time. We also analyzed the spatial distribution of labeled vacuoles in the same growth cones. The growth cones incubated in ferritin for $5 \mathrm{~min}$ had $37 \%$ of type III and IV vacuoles in the $\mathrm{P}$-domain, with the remaining $63 \%$ in the $\mathrm{C}$-domain. Growth cones from the $15 \mathrm{~min}$ incubation showed a similar distribution of these vacuoles: $33 \%$ were associated with

\begin{tabular}{|c|c|c|c|c|c|c|c|}
\hline $\begin{array}{l}\text { Incu- } \\
\text { bation } \\
\text { time } \\
\text { (min) }\end{array}$ & $\begin{array}{l}\text { \# } \\
\text { Cones }\end{array}$ & $\begin{array}{l}\text { Total } \\
\text { \# vacs }\end{array}$ & $\begin{array}{l}\text { Type } \\
\text { I }\end{array}$ & $\begin{array}{l}\text { Type } \\
\text { II }\end{array}$ & $\begin{array}{l}\text { Type } \\
\text { III }\end{array}$ & $\begin{array}{l}\text { Type } \\
\text { IV }\end{array}$ & $\begin{array}{l}\text { Ferritin } \\
\text { labeled }^{b}\end{array}$ \\
\hline 0 & 5 & 20 & 19 & 1 & 0 & 0 & - \\
\hline $1-2$ & 4 & 22 & 18 & 0 & 0 & 4 & $18 \%$ \\
\hline 5 & 6 & 19 & 8 & 1 & 8 & 2 & $53 \%$ \\
\hline 15 & 4 & 35 & 11 & 4 & 11 & 9 & $57 \%$ \\
\hline
\end{tabular}

All vacuoles in the indicated number of growth cones were categorized into one of four different types: type I, uniformly clear; type II, uniformly clear, but with a thin symmetrical electron-dense band underlying the organelle membrane; type III, mostly clear, but with very distinct patches contained within the vacuole and underlying the organelle membrane; and type IV, uniformly dark.

${ }^{\circ}$ Type III and IV vacuoles were never seen in control $(0 \mathrm{~min})$ growth cones and were therefore considered ferritin labeled. 
Figure 4. Stereo-pair micrographs of a complex, ruffling region at the leading edge of a growth cone. Vacuole-like organelles (arrowheads) were located both within and at the base of the ruffling lamellae. None of the organelles near ruffles had an orifice or appeared to be confluent with the plasmalemma. Instead, these organelles appeared to be trapped between ruffling lamellae. These images were acquired with the highvoltage electron microscope at Madison, WI.
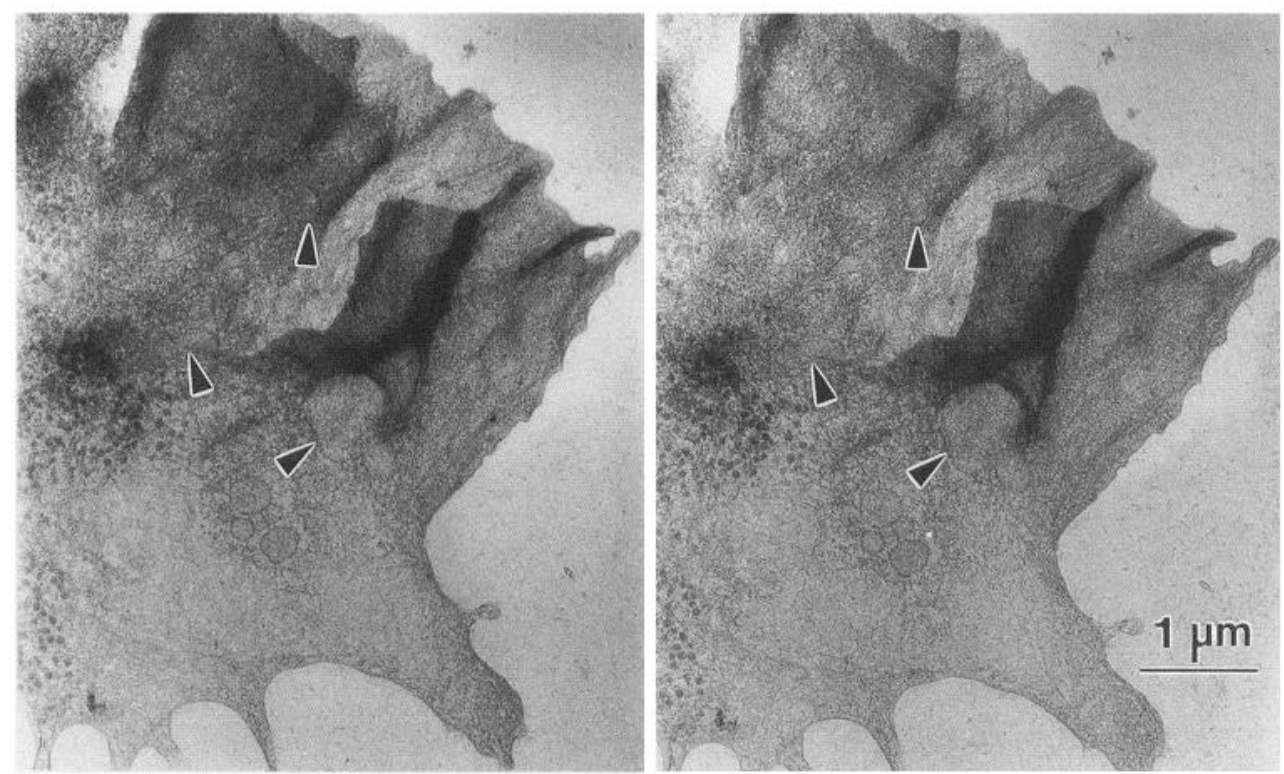

the P-domain while the remaining $67 \%$ were located in the C-domain.

To summarize thus far, the rapid-freezing EM results provide evidence that a set of vacuole-like intracellular membrane organelles interacts directly with the growth cone surface membrane. Rapid labeling of the organelles with exogenously applied ferritin suggests that the vacuoles retrieve membrane from the surface of growth cones. This does not, however, exclude the possibility that some vacuoles add membrane to the growth cone surface.

\section{Correspondence of vacuoles in the light and electron microscope}

Since the organelles that were labeled or interacting with the plasmalemma were all rather large in size $(\geq 150 \mathrm{~nm})$, we wondered whether they could be detected in the living state by highresolution video microscopy. Using video-enhanced contrastDIC microscopy, we found a number of large, vacuole-like organelles in living growth cones. Interestingly, these large organelles had a reversed shadowcast appearance; that is, the direction of the organelle's "shadow" was to the opposite side in comparison to all of the other organelles and structures in the growth cone (Fig. 6). Most of these organelles were found in the $\mathrm{C}$-domain, but some were observed in the P-domain. Although it was difficult to determine the size of these organelles precisely due to inflation of small objects (which is an inherent feature of the DIC image), they generally appeared larger than most other organelles, and some of them were up to $2 \mu \mathrm{m}$ in length. These organelles had spherical, ovoid, or elongated forms. Thus, the distribution, size, and appearance of the reverse shadowcast organelles suggested that they may correspond to the ferritinlabeled vacuoles observed in the EM.

To establish a direct relationship between the vacuoles observed in rapid-frozen whole-mounts and the reverse shadowcast organelles observed in video-enhanced DIC images, we performed LM and EM observations on the same growth cones. Because of the movement and transient nature of the reversed shadowcast organelles, it was not possible to use rapid freezing to prepare the whole-mounts for EM. There was too much time delay involved in removing the EM grid containing the cells from a closed chamber necessary for high-resolution DIC microscopy, and then mounting the grids for rapid freezing. For this reason we performed several experiments using direct chemical fixation with glutaraldehyde. Cultures with growth cones containing reverse shadowcast organelles were perfused with $0.25 \%$ glutaraldehyde while under direct observation with DIC microscopy. Although growth cones often showed some blebbing of surface membranes, there was no detectable change in the morphology of the reverse shadowcast organelles (data not shown). We therefore performed further experiments using two different techniques to label endocytotic organelles.

Ferritin labeling. First, we used cationized ferritin to label organelles using the same procedure as described for the rapidfrozen whole-mounts. After fixation with glutaraldehyde, growth cones on EM finder grids were visualized with video-enhanced DIC microscopy. Unfortunately, it was difficult to obtain a highquality DIC image using this procedure. The cationized ferritin coated not only the surface of the growth cone, but also the substratum. This coating was visible in DIC images as a speckled background. Sometimes aggregates of ferritin were present as highly birefringent spots that made detection of organelles difficult. (Labeling with rhodamine-conjugated cationized ferritin suffered from the same drawbacks: high background and aggregates). However, despite these problems we were able to resolve some of the larger reverse shadowcast organelles in several growth cones. When the same growth cones were observed in the EM, it was found that the large reverse shadowcast organelles corresponded to large vacuoles similar to those seen in rapid-frozen whole-mounts (Fig. 7). The internal surface of the vacuoles appeared to be coated with ferritin, although this was difficult to determine unambiguously in some cases because the ferritin density was not high in the large organelles and coating of the plasma membrane interfered with visualization of the organelle membrane.

Horseradish peroxidase labeling. Because of the problems associated with cationized ferritin labeling, an alternative method for visualizing endocytotic organelles was pursued using HRP histochemistry. HRP was added to the culture medium for 15 

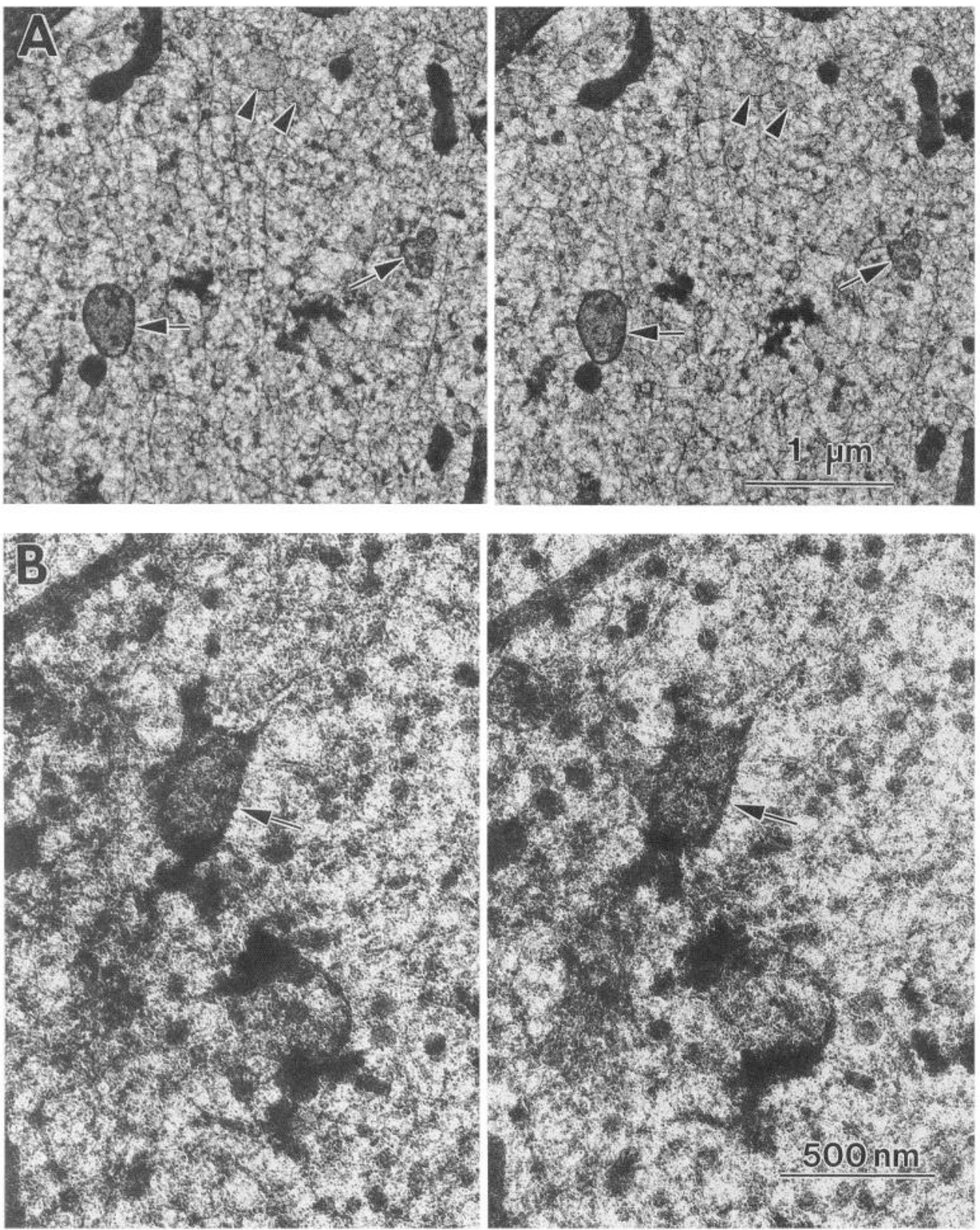

Figure 5. Some vacuoles in rapid-frozen growth cones could be labeled with exogenously applied ferritin. $A$, Portion of a growth cone in a culture incubated for $15 \mathrm{~min}$ in ferritin-containing medium. Individual particles (undetectable at this magnification) and clumps of ferritin coated the growth cone surface. Nevertheless, some vacuoles (arrows) appeared much darker in comparison to other vacuoles in the same field (arrowheads) or to all vacuoles in growth cones of unlabeled cultures (compare with Figs. 1-4). The darkest, elongated organelles located near the corners of the field are mitochondria, which appear dark in nontreated growth cones as well. $B$, High-magnification view of a labeled organelle (arrow) from a different growth cone incubated for $15 \mathrm{~min}$. The small $(70 \mathrm{~nm})$ dark spheres are dense-core vesicles, which are often found in the C-domain of these growth cones (compare with Figs. 1 $B, 4$ ). min and then washed out for 3 min prior to fixation with glutaraldehyde. Growth cones were then imaged using video-enhanced DIC microscopy. This labeling procedure allowed detection of smaller reverse shadowcast organelles than in cells labeled with ferritin because there was essentially no contamination by HRP background signal. The DIC images were still slightly compromised because a longer-working-distance lens with lower numerical aperture was needed to image cells grown on EM grids and, in addition, diffraction of light from the EM grid bars and formvar film affected the image quality. Nevertheless, we were able to obtain reasonably good images of reverse shadowcast organelles in 11 growth cones. After DIC images were obtained, the cultures were processed for HRP histochemistry using the Hanker-Yates procedure (Hanker et al., 1977). The growth cones were again imaged in the LM using the DIC configuration but without the Wollaston prism of the objective lens. This allowed easy detection of the dark HRP reaction product and yielded a low-contrast image of the growth cone for comparison to the DIC image (Figs. 8, 9). After this set of images was obtained, the cultures were further processed for EM. The growth cones that had been imaged by LM were reidentified at the EM level using the finder grid location as a guide. We successfully identified all 11 growth cones using this procedure.

Comparison of the LM and EM images of individual growth cones indicated that reverse shadowcast organelles always corresponded to vacuoles at the EM level and most, but not all, of these vacuoles were labeled with HRP reaction product (Figs. 8-10, Table 2). Sixteen percent of the reverse shadowcast organelles that were labeled with HRP were located in the P-domain, while the remaining $84 \%$ were in the $\mathrm{C}$-domain. The relatively low percentage within the $\mathrm{P}$-domain (compared to the percentage seen with ferritin labeling) probably resulted from the longer washout period $(3 \mathrm{~min}$ vs. $<1 \mathrm{~min})$. When we ex- 


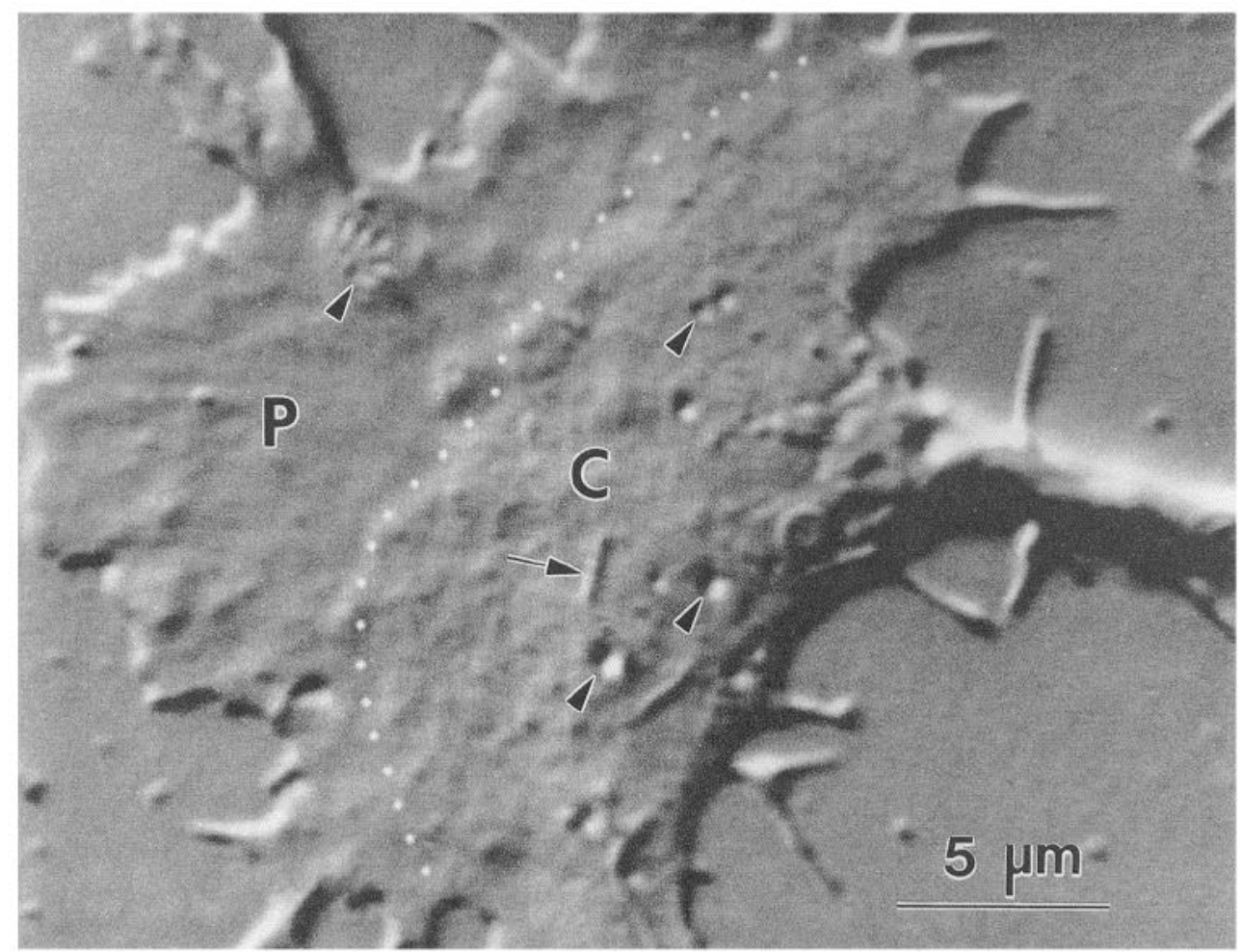

Figure 6. Vacuole-like organelles (arrowheads) had a conspicuous reverse shadowcast appearance in video-enhanced DIC images. DIC microscopy is a form of interference microscopy that produces a shadowcast relief appearance of cellular constituents. Virtually all cell constituents in a given field have the same shadow orientation. Most cell components are, for example, bright on the upper left side and dark on the lower right side. However, sometimes cell constituents have a reverse shadowcast appearance (they are bright on the side opposite that of most other elements). The shadowcast appearance is normally a result of differential retardation of light through two adjacent pathways along a specific shear direction. For example, at the edge of a cell, light that travels through the cytoplasm is recombined with light that does not travel through cytoplasm, resulting in constructive or destructive interference (bright or dark regions), depending on the direction of shear set by the Wollaston prism (Allen et al., 1981). The occurrence of organelles with a reverse shadowcast appearance probably results from a lesser degree of retardation of light through these organelles relative to other organelles. This is consistent with the idea that the material within these organelles has a lower index of refraction than that of other organelles or the surrounding cytoplasm. In this growth cone, there are several reverse shadowcast organelles (arrowheads) in both the $\mathrm{C}$-domain $(C)$ and $\mathrm{P}$-domain $(P)$. Compare these organelles with a mitochondrion (arrow), which has the usual shadowcast relief.

amined the distribution of the reverse shadowcast organelles that were not labeled, four of nine $(44 \%)$ were in the P-domain, suggesting that they had probably formed during the 3 min HRP washout (because most P-domain organelles have a very short lifetime; see Fig. 16). The remaining five unlabeled organelles that were located in the $\mathrm{C}$-domain may have also formed during the washout, or they may have been present prior to the HRP incubation. In addition, it was apparent that HRP reaction product could be detected by LM where no obvious reverse sha-

\begin{tabular}{lllll}
\hline Table 2. & HRP labeling of reverse shadowcast organelles \\
& & & \\
\# & $\begin{array}{l}\text { \# Reverse } \\
\text { Growth } \\
\text { shadow- } \\
\text { cones }\end{array}$ & $\begin{array}{l}\text { cast } \\
\text { +HRP }\end{array}$ & $\begin{array}{l}\text { - HRP } \\
\text { (LM) }\end{array}$ & $\begin{array}{l}\text { +HRP } \\
\text { (EM) }\end{array}$ \\
\hline 11 & 45 & $36^{b}(80 \%)$ & $9(20 \%)$ & $46^{b}(62 \%)^{c}$
\end{tabular}

${ }^{a}$ Data were taken from growth cones grown on EM grids that were incubated in HRP for $15 \mathrm{~min}$, rinsed for $3 \mathrm{~min}$, chemically fixed, and then imaged sequentially by DIC, modified DIC (without Wollaston prism), and whole-mount EM

${ }^{h}$ Ten additional HRP-positive spots were also detected by LM, but they did not correspond to detectable reverse or normal shadowcast organelles in the DIC image. These were not included in the LM total. However, they did correspond to HRP-positive vacuoles as observed with EM, accounting for the difference in HRP-positive vacuoles between LM and EM.

c Percentage of the total number of vacuoles seen by EM. dowcast organelle could be seen by DIC (Figs. 8, 9; Table 2). EM examination revealed vacuoles containing reaction product at these sites, but these organelles were usually situated adjacent to other organelles, and they tended to be small. Thus, the static DIC images did not always reveal organelles involved in the uptake of HRP if the organelles were particularly small, especially in areas where they were tightly packed together. By EM, the HRP-labeled vacuoles represented $62 \%$ of the total number of vacuoles. In two growth cones, HRP reaction product was also found within vacuoles that appeared to have an orifice communicating with the extracellular environment (e.g., Fig. $10 \mathrm{~B}$ ), suggesting that these organelles were probably involved in exocytosis at the time of fixation.

These results establish that reverse shadowcast organelles in DIC images correspond to intracellular membrane vacuoles, many of which are derived from the surface membrane.

\section{Video microscopy of vacuole dynamics}

To examine the movement and dynamics of growth cone vacuoles directly, we followed reverse shadowcast organelles in living growth cones using real-time DIC video microscopy. We used real-time ( $33 \mathrm{msec}$ frame intervals) rather than time-lapse video microscopy in order to capture any rapid, transient events. Of the living growth cones we analyzed in detail, $73 \%$ (11 of 15) had at least one reverse shadowcast organelle during the 

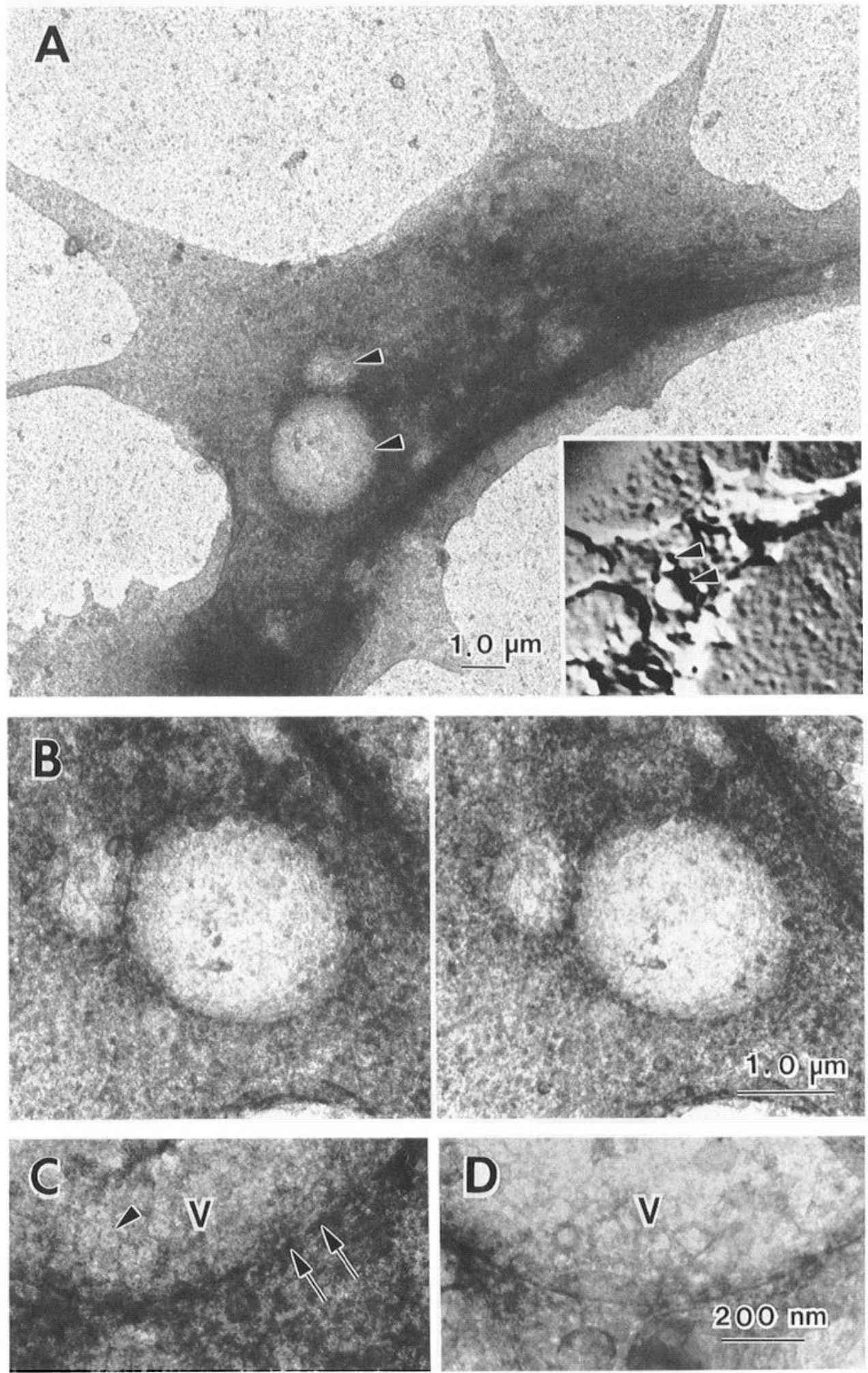

Figure 7. Some of the large reverse shadowcast organelles in DIC images correspond to ferritin-labeled vacuoles in the EM. $A$, Growth cone that was incubated in cationized ferritin for 15 min, washed, and then fixed with glutaraldehyde. The ferritin coated the cell surface and substratum and appears as dark aggregates of particles in the wholemount EM image. Two vacuoles that were identified as reverse shadowcast organelles by DIC microscopy (inset) are evident (arrowheads). B, A highermagnification stereo view of the vacuoles indicated in $A$. Both appear as closed vesicles and have a relatively dark rim with dark patches characteristic of organelles labeled with ferritin. $C, \mathrm{~A}$ portion of the larger vacuole shown at higher magnification. The dark rim of the vacuole is indicated (double arrows). Dark ferritin particles can be seen coating the vacuole membrane (arrowhead). $D$, A portion of a different vacuole (that was identified as a reverse shadowcast organelle by DIC microsccopy) from an unlabeled culture is shown for comparison. period of observation (average observation period, $19 \mathrm{~min}$; range, $10-60 \mathrm{~min}$ ). Growth cones typically contained from one to five organelles with a reverse shadowcast appearance, although the number of such organelles in a single growth cone ranged from 0 to 20 at any given time.

Our video observations indicated that the reverse shadowcast organelles were formed or first appeared at various locations throughout the growth cone. These organelles spontaneously appeared at some sites of ruffling at the leading edge of the growth cone (Fig. 11). Although often a single large organelle emerged from the ruffling region, sometimes a cluster of smaller organelles appeared. All the organelles then moved in a centripetal direction toward the growth cone base at a rate of about $1 \mu \mathrm{m} / \mathrm{min}$. This is comparable to the rate of centripetal movement of materials in growth cones described previously (Forscher et al., 1987; Forscher and Smith, 1988; Dailey and Bridgman, 
Figure 8. Reverse shadowcast organelles can also be labeled with HRP. $A$, DIC image of a growth cone on an EM grid that was incubated in HRP for 15 min prior to a $3 \mathrm{~min}$ wash and then fixed with glutaraldehyde. Two reverse shadowcast organelles can be seen at the base of the growth cone (arrowheads). B, An LM image of the same growth cone shown in $A$ after histochemical production of HRP reaction product. The image was obtained without the Wollaston prism in place to allow easy visualization of the dark reaction product. Both reverse shadowcast organelles are labeled (arrowheads). A third vesicle not seen in the DIC image is also labeled in the proximal portion of the neurite. $C$, A whole-mount EM image of the same growth cone shown in $A$ and $B$. The two labeled vacuoles can be seen (arrowheads). The reaction product tends to line the inner surface of the vacuole membrane. See Figure $10 \mathrm{~A}$ for higher-magnification stereo-pair images of this region.
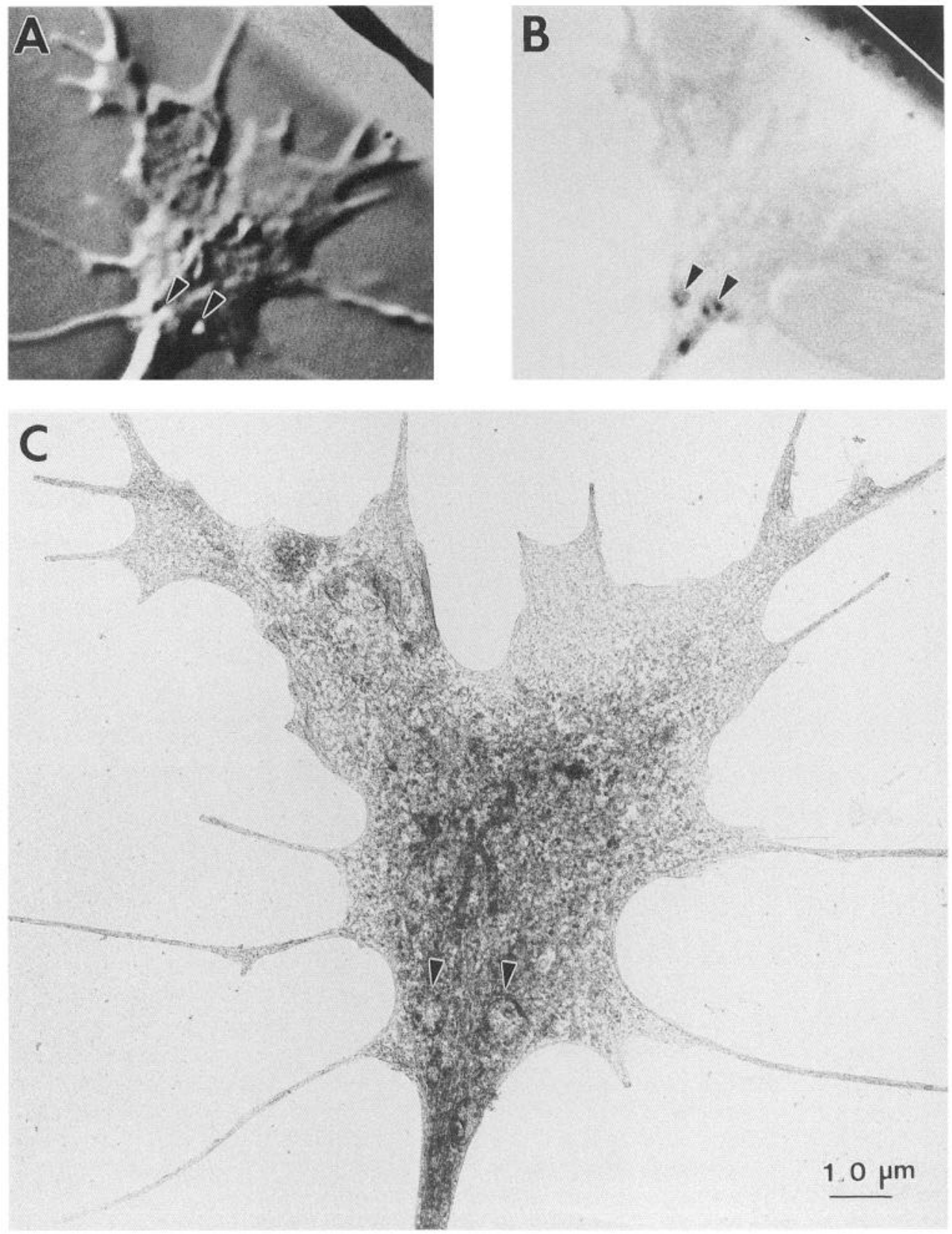

1989). Although some of the centripetally moving organelles were transported to the C-domain, many of the organelles disappeared from the video image while in transport through the P-domain. Individual organelles of a cluster were observed to disappear independently (Fig. 11).

Ruffling-like activity was also observed near the center or midbody of many growth cones in our cultures. This activity was manifested by dynamic cytoplasmic/membrane protrusions that erupted from, and then collapsed back onto, the growth cone surface. Reverse shadowcast organelles emerged from these sites of midbody ruffling (Fig. 12), and these organelles then moved centripetally until they reached the large mass of organelles that occupied the C-domain. Thus, a consistent finding was that organelles that emerged in the P-domain at sites of ruffling, whether at the leading edge or midbody, invariably moved toward the base (C-domain) of the growth cone. We never ob- served vacuoles moving anterogradely in a directed manner from the C-domain into the P-domain filopodia or lamellipodia.

Reverse shadowcast organelles also spontaneously appeared within the C-domain. The organelles sometimes first became apparent near the edge at the base of the growth cone, but they also simply "popped into view" in the center of the C-domain organelle mass. No ruffling activity was observed when these organelles first appeared in the C-domain. Those organelles that popped into view were first evident as very small spherical objects that moved around in a Brownian-like manner. Subsequently, they would slowly grow in size over time (Fig. 13A). Growth of organelles was evident even as they moved around at the base of the growth cone. In contrast, organelles formed in the P-domain by ruffling at the leading edge or midbody did not exhibit growth over time. However, some of these organelles appeared to fuse together (Fig. 13B). 

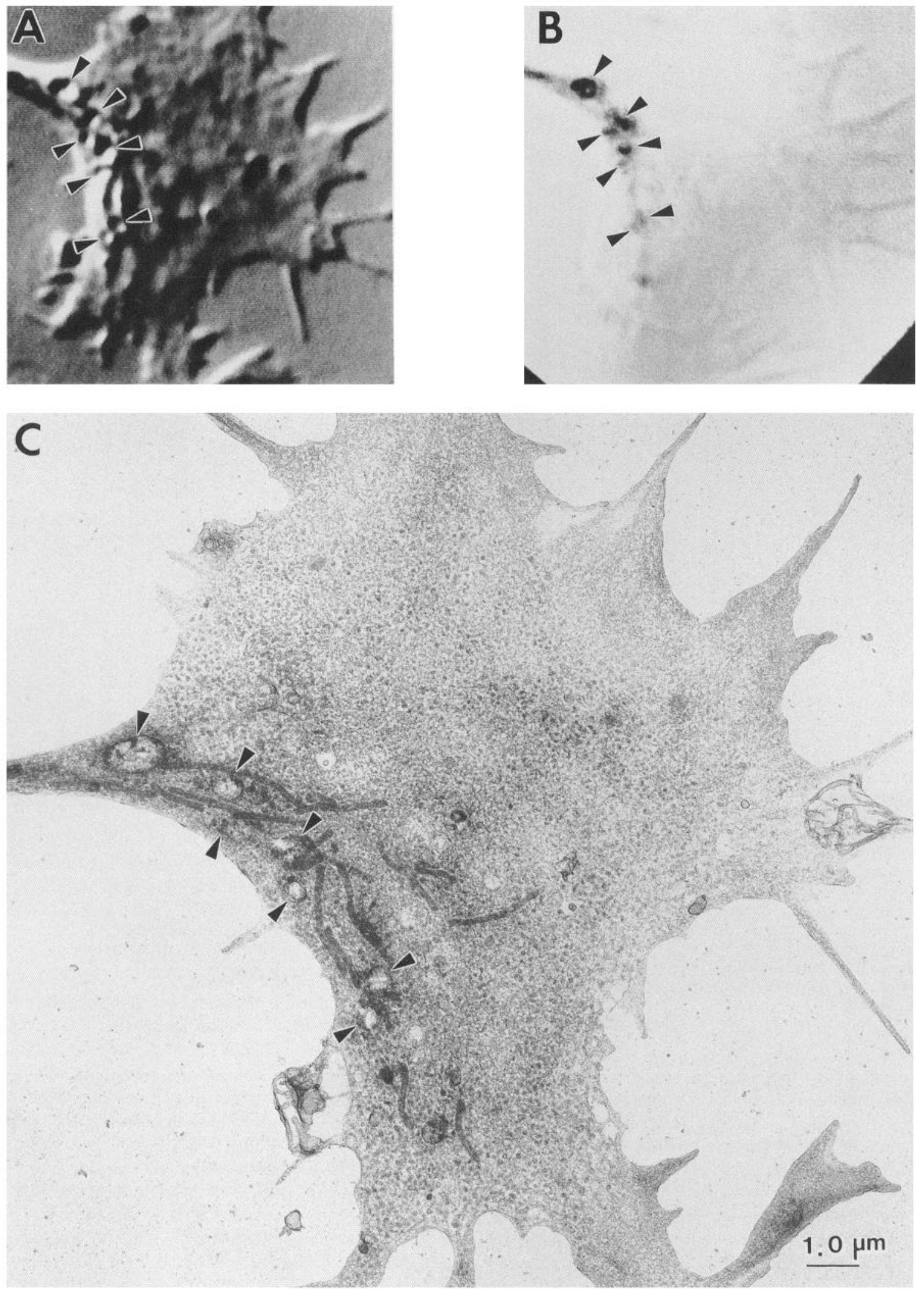

Figure 9. Correspondence of reverse shadowcast organelles and HRP. A, DIC image of a growth cone grown on an EM grid that was incubated with HRP for $15 \mathrm{~min}$, washed for $3 \mathrm{~min}$, and then glutaraldehyde fixed. An especially large number of reverse shadowcast organelles can be seen (arrowheads). B, An image (without Wollaston prism) of the same growth cone shown in $A$ after histochemical production of HRP reaction product. All the detectable reverse shadowcast organelles seen in $A$ contain the dark HRP reaction product. At least one additional vacuole that was not detected by DIC is also labeled with HRP. $C$, Whole-mount EM image of the same growth cone shown in $A$ and $B$. Vacuoles containing HRP reaction product (arrowheads) correspond to the reverse shadowcast organelles seen by DIC microscopy. See Figure $10 B$ for higher-magnification stereo-pair images. 


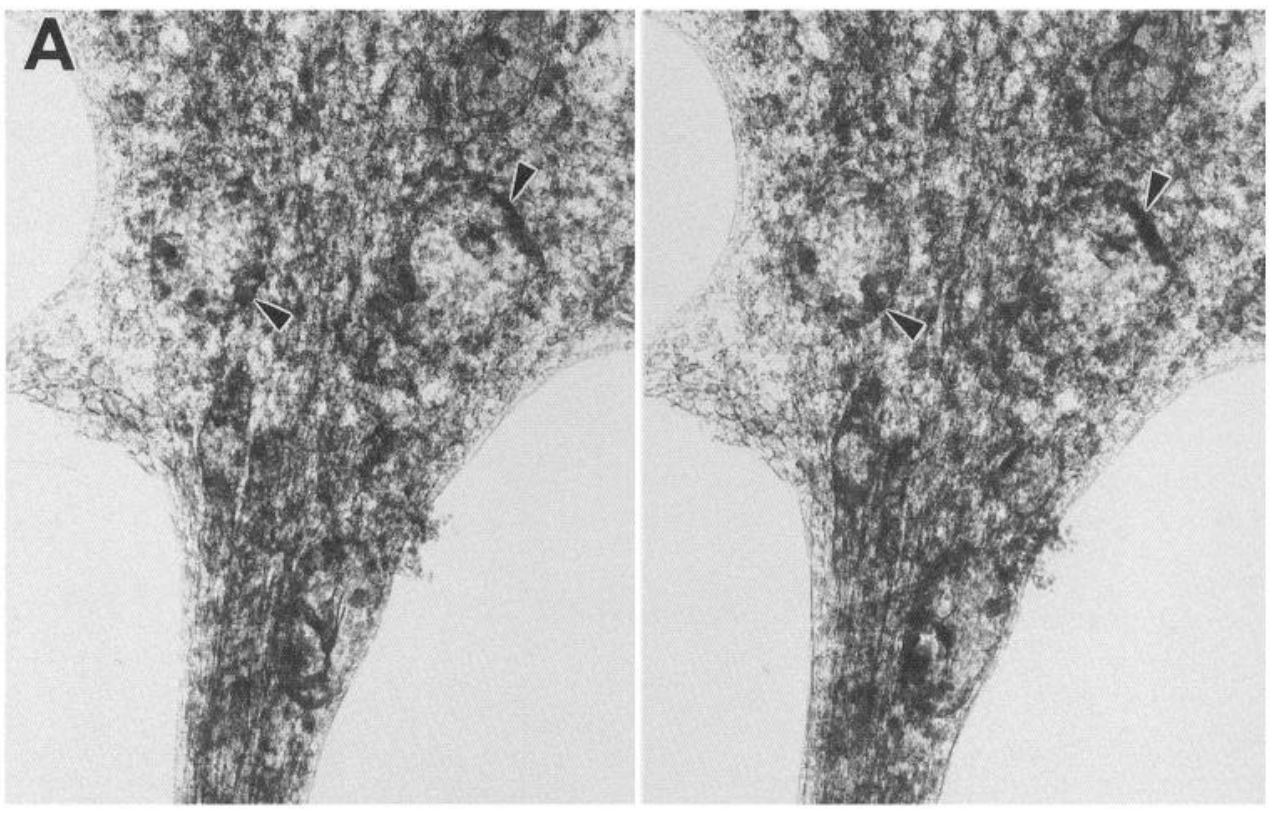

Figure 10. Stereo-pair EM images of HRP-labeled vacuoles. $A$, A highermagnification stereo view of the HRPlabeled vacuoles from the growth cone shown in Figure 8. Patches of HRP reaction product (arrowheads) line the inner surface of the vacuole membrane. $B$, Stereo-pair images of the HRP-labeled vacuoles (arrowheads) from the growth cone shown in Figure 9. One vacuole (double arrowheads) that contains reaction product appears also to be fused with the plasma membrane and open to the extracellular space through a distinct orifice. This may indicate exocytosis (membrane recycling) because HRP was washed off $3 \mathrm{~min}$ prior to fixation.
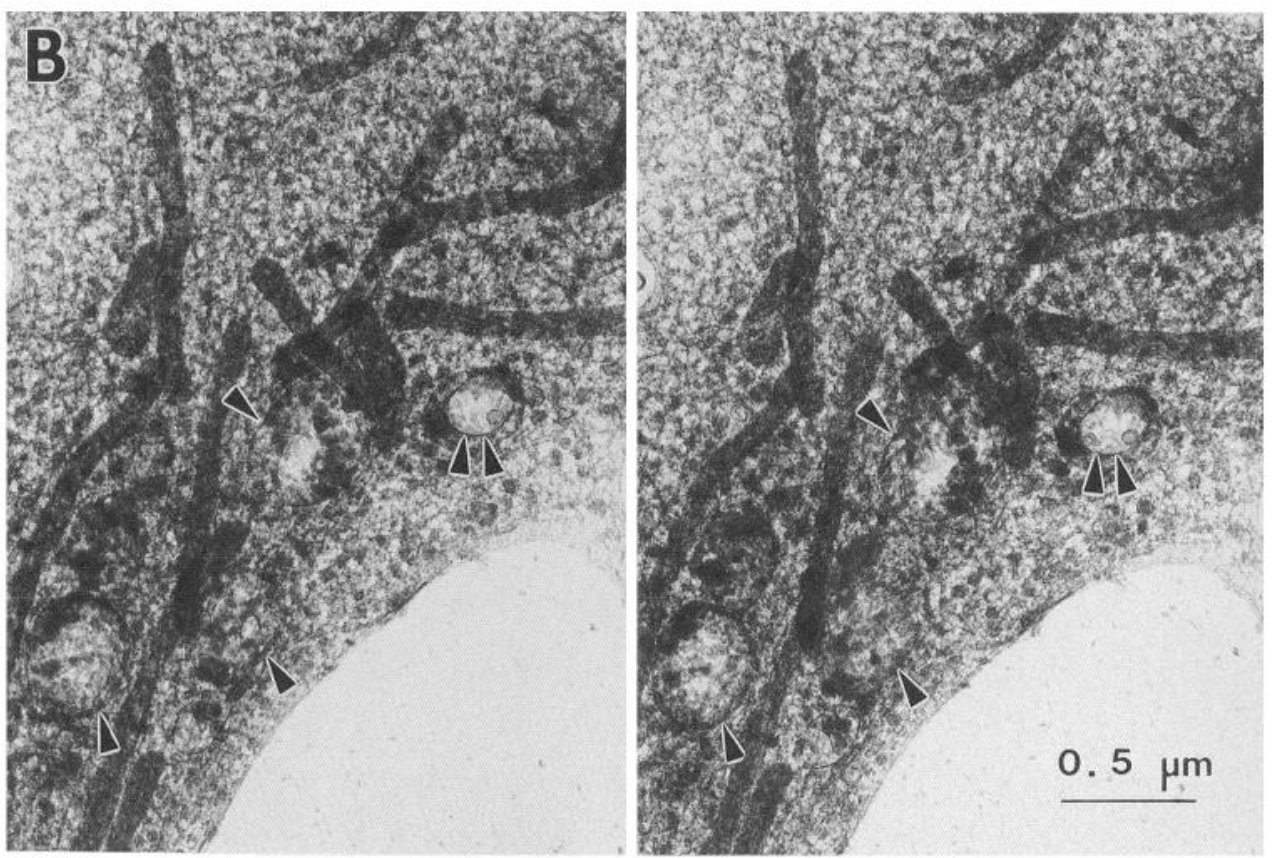

Although some of the reverse shadowcast organelles were transported down the neurite, most remained within the growth cone until they disappeared from the video image. Organelles that first emerged at ruffles sometimes disappeared very near their site of origin in the P-domain (Fig. 11). Some, however, were transported to the C-domain, where they subsequently disappeared. We often observed organelles shrinking before they disappeared, and this was most evident when the organelle was very large (Fig. 14). The process of organelle shrinking and disappearance in time-lapse sequences can be likened to a deflating balloon. We did not see stepwise shrinking, or cycles of shrinking and growing. We observed only one incident in which a reverse shadowcast organelle appeared to vesiculate. Even in this case the resultant vesicles had a reverse shadowcast appearance until they disappeared shortly after being formed (Fig. $15)$.
To compare the lifetime of organelles in different growth cone regions, we calculated from real-time video sequences the total length of time that individual organelles were visible. Organelles formed at sites of ruffling were generally visible for less than 5 min, whereas most of the organelles that first appeared in the $\mathrm{C}$-domain were visible for longer than $10 \mathrm{~min}$. At least some of the C-domain organelles were visible for over $20 \mathrm{~min}$. Thus, organelles formed at the growth cone base had a significantly longer lifetime than those formed near P-domain ruffles (Fig. 16). A summary of the distinctive, domain-specific behaviors exhibited by vacuoles is given in Table 3 .

\section{Discussion}

Growth cones both in vivo and in vitro are characterized by a rather large number and variety of intracellular membrane organelles (reviewed in Gordon-Weeks, 1988). It is suspected that 

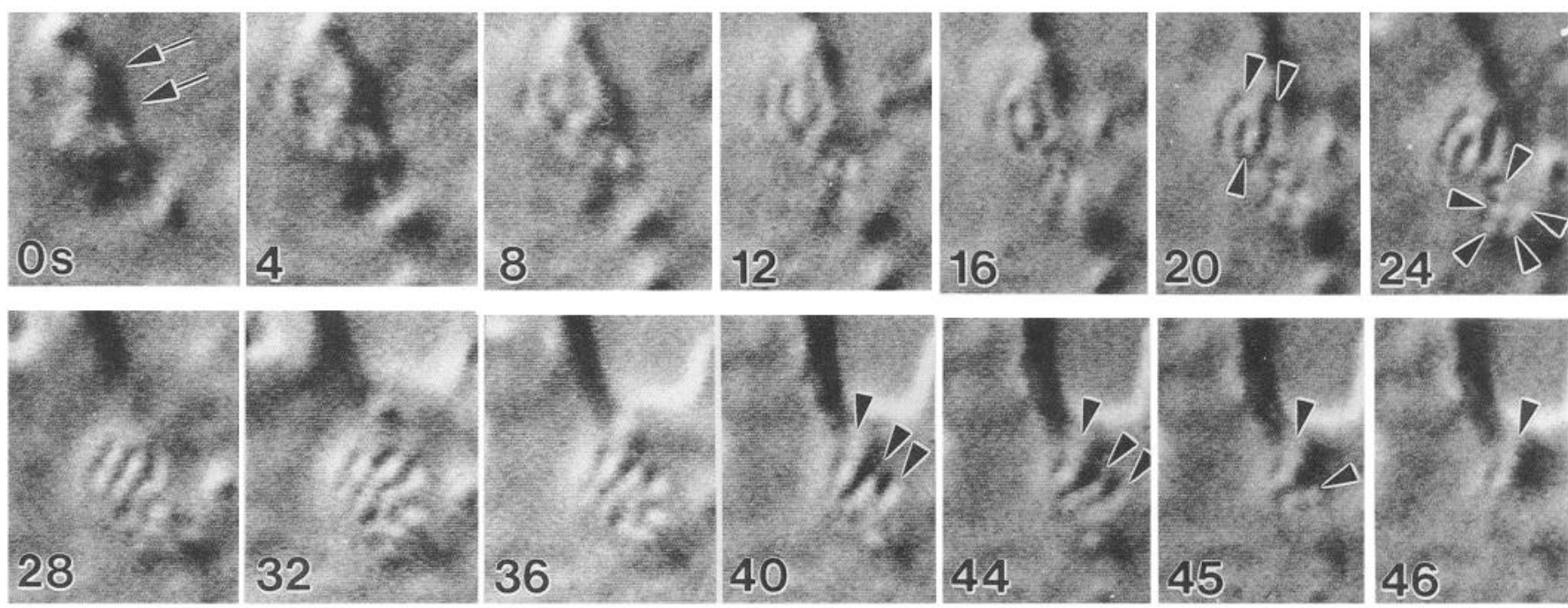

Figure 11. Intracellular membrane organelles with a reverse shadowcast appearance emerged at sites of ruffling near the leading edge and moved centripetally toward the growth cone base (same growth cone as Fig. 6). A cluster of organelles was formed at a site of ruffling (double arrows at 0 $\mathrm{sec}$ ). Elongated (arrowheads at $20 \mathrm{sec}$ ) and spherical (arrowheads at $24 \mathrm{sec}$ ) organelles moved together from upper left to lower right, that is, from the leading edge of the growth cone toward the C-domain. Most of the organelles rapidly disappeared from view. For example, note at the last three time points that two of the elongated organelles disappeared sequentially. Real-time imaging indicated that disappearance was not due to rapid translocation away from this area, nor was vesiculation apparent. The field is $3 \mu \mathrm{m}$ wide, and time is indicated in seconds.

these organelles may play an important role in supporting the high degree of surface membrane dynamics that accompany normal growth cone activity and neurite elongation. However, surprisingly little is known about the fundamental mechanisms and regulation of surface membrane addition and retrieval at the growth cone. We have begun to investigate these issues by applying techniques that permit direct viewing of some dynamic membrane events.

\section{Interaction of organelles with the plasmalemma: membrane retrieval}

Our goal was to identify intracellular membrane organelles that were physically interacting with the plasmalemma, thereby indicating possible membrane addition or retrieval events. The rapid-freezing and freeze-substitution protocol enabled us to capture some interactions between membrane vacuoles and the plasmalemma, presumably because membranes are "fixed" more rapidly and suffer less distortion when prepared in this manner (Knoll et al., 1987; Sitte et al., 1987). We used whole-mount EM because many more growth cones could be observed than with thin-section EM, and this increased the likelihood of viewing fleeting membrane events. Moreover, the whole-mount provides panoramic views of growth cones similar to that of the LM, and this greatly facilitates identification of individual organelles in both the LM and EM. The whole-mount view also enables one to surmise the motile activity of different growth cone regions at the time of "fixation" based on general morphology and local ultrastructure. Previous work has shown that protruding, stable, and retracting regions of growth cones (Bunge, 1973; Tosney and Wessells, 1983) and other motile cells (e.g.,
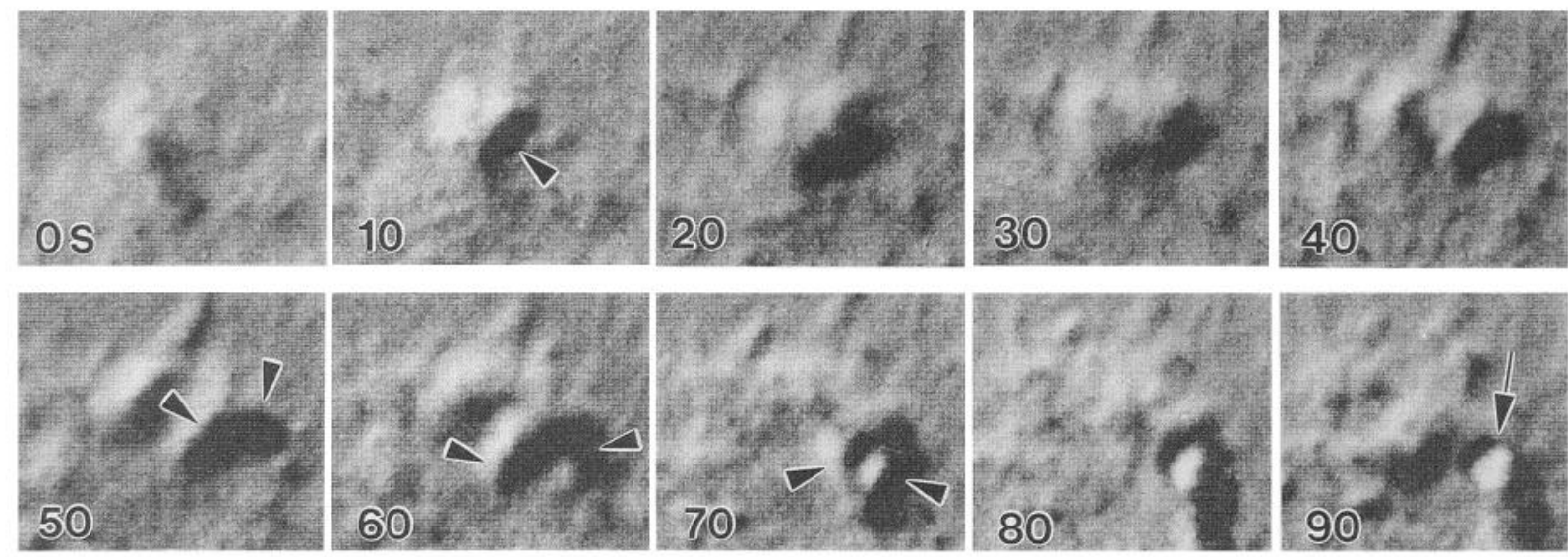

Figure 12. Formation of reverse shadowcast organelles by ruffling activity at the growth cone midbody (i.e., the transition zone between the Cand P-domains). Time-lapse sequences indicated that thickened mounds of cytoplasm (arrowheads) grew and extended linear processes that appeared to protrude from the body of the growth cone. The arcs sometimes merged together or collapsed back onto the growth cone surface, forming intracellular organelles that had a reverse shadowcast appearance (arrow). This motile activity was designated "midbody ruffling." The images are at $10 \mathrm{sec}$ intervals, and the field width is $5 \mu \mathrm{m}$. 

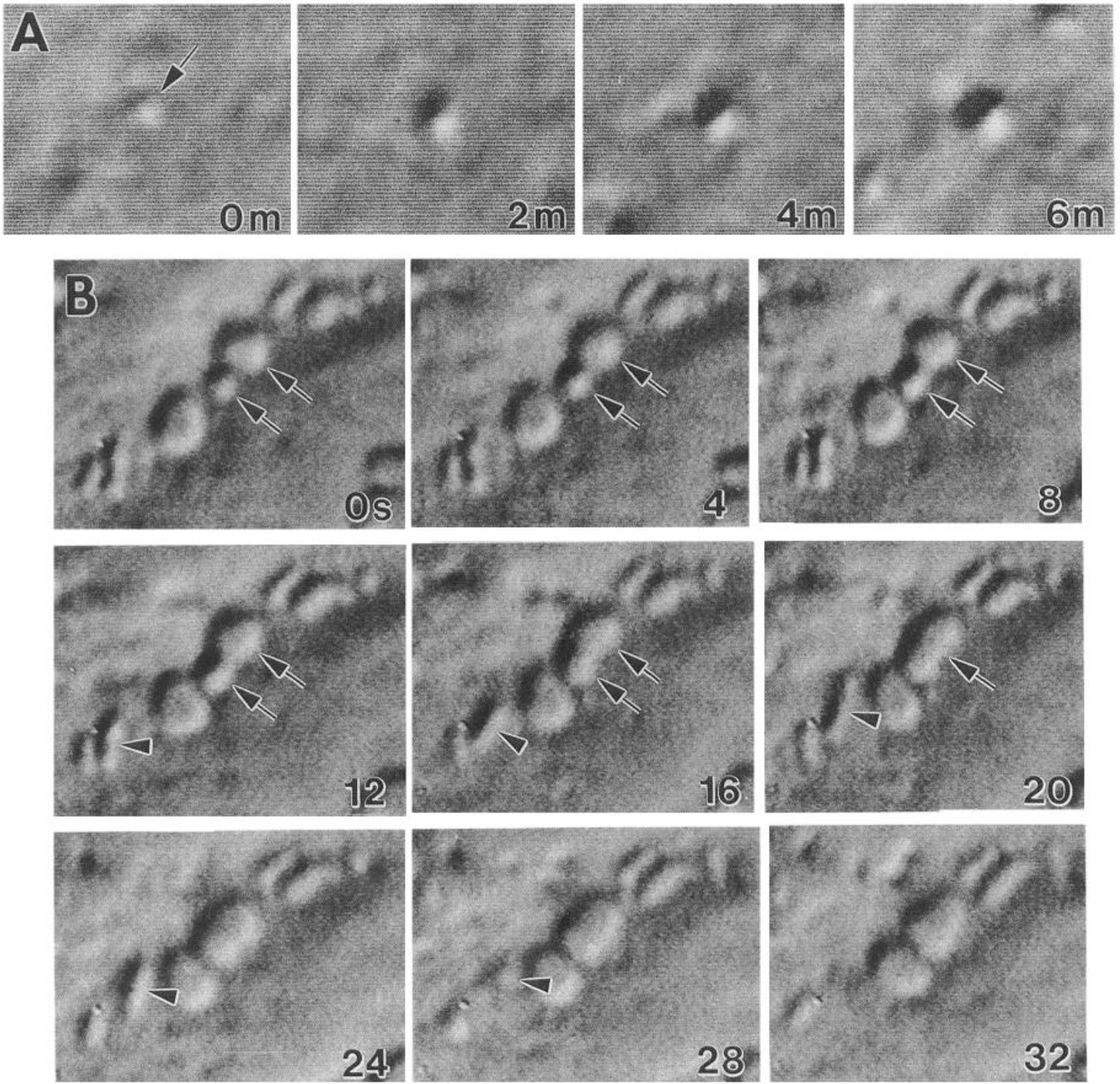

Figure 13. Some reverse shadowcast organelles grew in size over time. A, Organelles in the C-domain grew in the absence of visible organelle fusion. Note the slow growth in size of a single organelle, which exhibited Brownian-like movements while slowly translocating a net distance of several microns within the $\mathrm{C}$-domain. Images are at $2 \mathrm{~min}$ intervals, and the field width is $2.5 \mu \mathrm{m}$. $B$, Organelles in the P-domain did not grow in size, except by fusion with other vacuoles. In this P-domain field, two organelles (arrows) apparently fused together, and another organelle (arrowhead) shrank and disappeared. The images are at $4 \mathrm{sec}$ intervals; field width is $5 \mu \mathrm{m}$.

\section{Table 3. Domain-specific characteristics of reverse shadowcast} organelles $^{a}$

\begin{tabular}{lllll} 
Location $^{b}$ & $\begin{array}{l}\text { Local motile } \\
\text { activity }^{c}\end{array}$ & $\begin{array}{l}\text { Organelle } \\
\text { movement }^{2}\end{array}$ & $\begin{array}{l}\text { Organ- } \\
\text { elle } \\
\text { growth }\end{array}$ & $\begin{array}{l}\text { Organelle } \\
\text { lifespan }\end{array}$ \\
\hline $\begin{array}{l}\text { P-domain } \\
\text { C-domain }\end{array}$ & $\begin{array}{l}\text { Ruffling } \\
\text { Quiescent/retracting }\end{array}$ & $\begin{array}{l}\text { Centripetal }^{d} \\
\text { Brownian-like }\end{array}$ & $\begin{array}{l}\text { No } \\
\text { Yes }\end{array}$ & $>5 \mathrm{~min}$ \\
\end{tabular}

a This table summarizes general characteristics of organelles with a reverse shadowcast appearance in DIC video images. See Results for details.

${ }^{b}$ Indicates the domain in which the organelles first appeared in the video image. "P-domain" refers to the actin-rich peripheral region and includes the leading edge and midbody of the growth cone. "C-domain" refers to the microtubuleand organelle-rich central region at the base of the growth cone (see Bridgman, 1992).

c Refers to the predominant local motile activity at the time when the organelles first appeared in the video image.

${ }_{d} \sim 1 \mu \mathrm{m} / \mathrm{min}$
Buckley and Porter, 1967; Buckley, 1974) in culture have stereotypical ultrastructural characteristics that correspond to local motile activities. Protruding regions have a dense cortical meshwork of microfilaments and typically lack membrane organelles. Quiescent (stable) areas have a looser three-dimensional lattice of filaments, and many filaments have their long axis parallel to the contour of the growth cone margin. These regions also contain microtubules and a variety of intracellular membrane organelles. Retracting regions of glutaraldehyde-fixed growth cones were shown to contain strings of vesicles in filament-free space (Tosney and Wessells, 1983), but since these features were not found in cultured growth cones prepared by rapid-freezing methods (Rees and Reese, 1981; Bridgman and Dailey, 1989), they may simply indiciate glutaraldehyde-sensitive membrane lability. In practice, actively retracting areas may be difficult to distinguish from quiescent regions in rapid-frozen specimens.

Based on these morphological criteria and the labeling ex- 

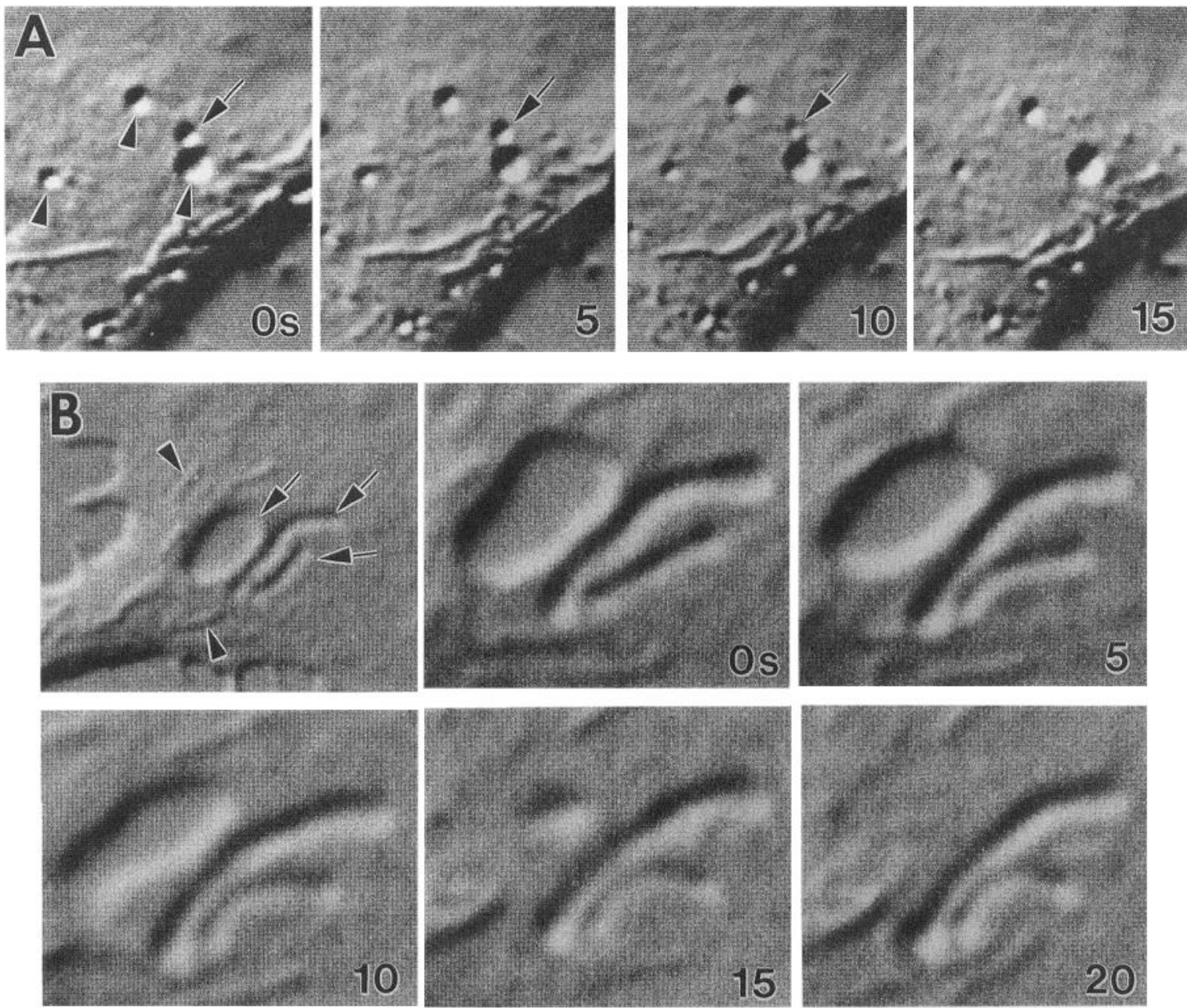

Figure 14. Reverse shadowcast organelles within the C-domain shrank and rapidly disappeared. $A$, This field near the neck of the growth cone contains several reverse shadowcast organelles (arrowheads), one of which shrank and disappeared (arrow). Images are at 5 sec intervals; field width is $4 \mu \mathrm{m}$. $B$, Some unusually large reverse shadowcast organelles (arrows) were found at the base of this growth cone. Contrast their size and appearance with that of nearby mitochondria (arrowheads), which are smaller and have the usual shadowcast appearance. Higher-magnification, time-lapse sequence shows the rapid shrinkage and disappearance of the large oval organelle (compare the size and shape with Fig. $3 B$ ). During this sequence the elongated organelles made short forward and rearward excursions, as if momentarily undergoing anterograde and retrograde directed transport. Several minutes later, the smaller organelle was transported retrogradely down the neurite. Images are at 5 sec intervals. The low-mag field width is $10 \mu \mathrm{m}$, and the high-mag field width is $5 \mu \mathrm{m}$.

periments, many of the organelle- and surface-membrane events we observed by whole-mount EM probably represent retrieval of surface membrane. We found intracellular membrane organelles that were confluent with the plasmalemma in areas that had ultrastructural characteristics expected of quiescent or retracting regions, and it seems likely that membrane events at these sites represent membrane retrieval. That membrane is retrieved by these organelles is consistent with the finding that a morphologically identical set of organelles could be rapidly labeled with exogenously applied ferritin particles or HRP. In fact, up to $62 \%$ of the total vacuole population could be labeled with 15 min incubations, indicating that a majority of these organelles are involved in membrane uptake.

Other organelles besides the vacuoles may also be involved in surface membrane uptake. Previous thin-section EM studies of tracer uptake into rat or chick sympathetic growth cones in culture have indicated that a wide variety of organelle types can be rapidly labeled with HRP or ferritin, including coated and uncoated vesicles, branched and unbranched membranous tu- bules, cup-shaped structures, small vacuoles, and clusters of subplasmalemmal vesicles (Birks et al., 1972; Bunge, 1977). Whether some of these structures are artificially generated during aldehyde fixation is debated, but even in directly frozen chick tectal growth cones, cationized ferritin labeled coated pits, coated vesicles, smooth vesicles, and vacuoles (Cheng and Reese, 1987). We focused our attention on the vacuoles because only with these organelles did we observe a direct interaction with the plasma membrane. It is possible that other, small organelles were labeled as well, but it is difficult to identify the label unequivocally in small vesicles in the complex whole-mount EM images. Nevertheless, the correlated LM and EM images (Figs. 8,9 ) suggested that most of the internalized HRP corresponded to the vacuoles.

With regard to the sites of membrane uptake, most of the ferritin- and HRP-labeled vacuoles we observed were located in the $\mathrm{C}$-domain, and not near the leading edge (P-domain). This was also true of the organelle- and surface-membrane interactions observed in rapid-frozen EM specimens. In contrast, 

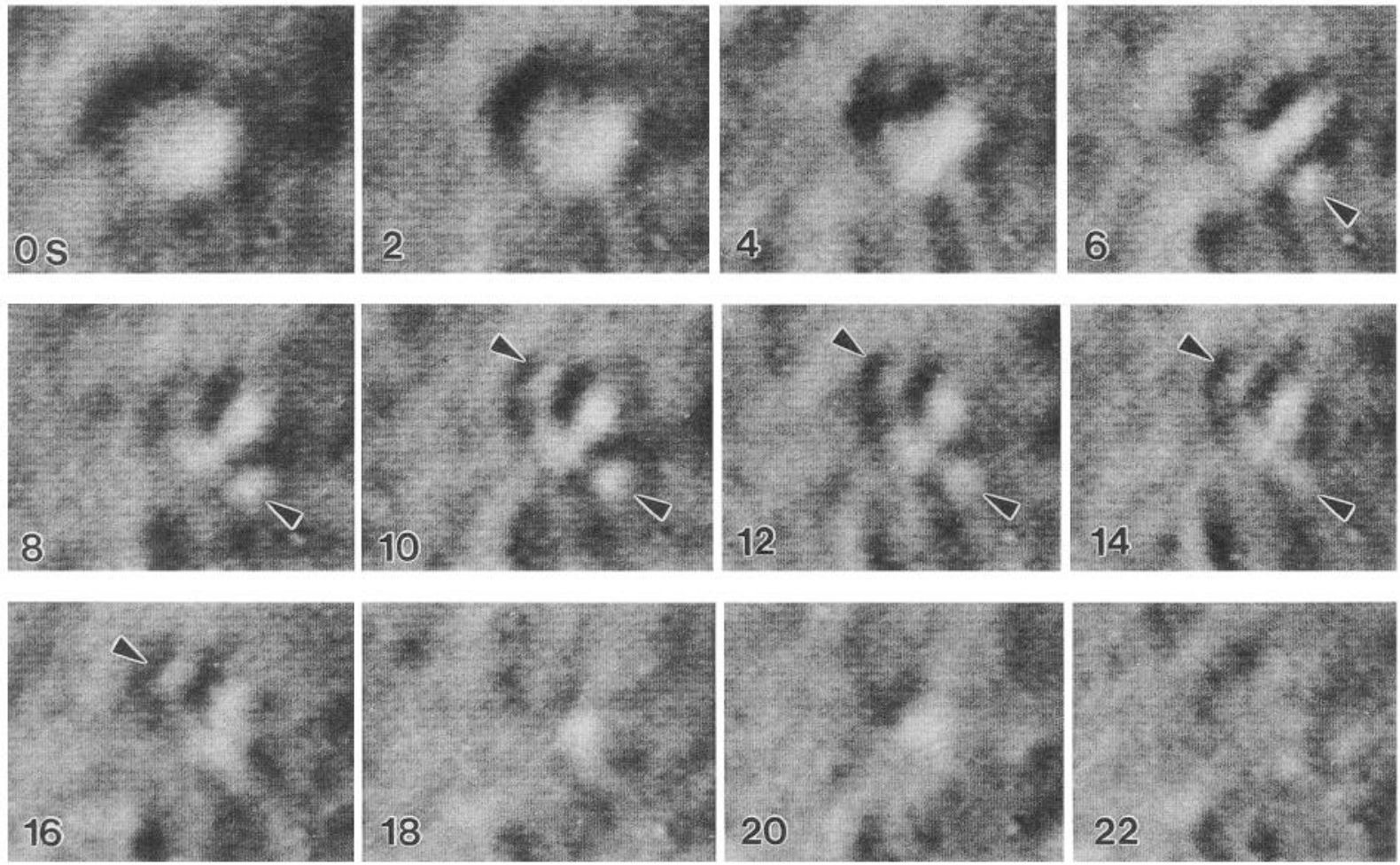

Figure 15. Reverse shadowcast organelles rarely vesiculated. This was the only example we observed of an organelle that subdivided into smaller vesicles (arrowheads), each of which subsequently disappeared. Note that even the smaller, transient vesicles had a reverse shadowcast appearance. Images are at $2 \mathrm{sec}$ intervals, and the field width is $2.5 \mu \mathrm{m}$.

Bunge (1977) concluded that endocytosis of ferritin or HRP by cultured rat sympathetic neurons occurs primarily at the leading edge rather than at the base of growth cones (although the largest HRP-labeled organelles appeared to be near the base; cf. Fig. 22 in Bunge, 1977). The different observations may possibly be

\section{LIFETIME OF REVERSE SHADOWCAST ORGANELLES}

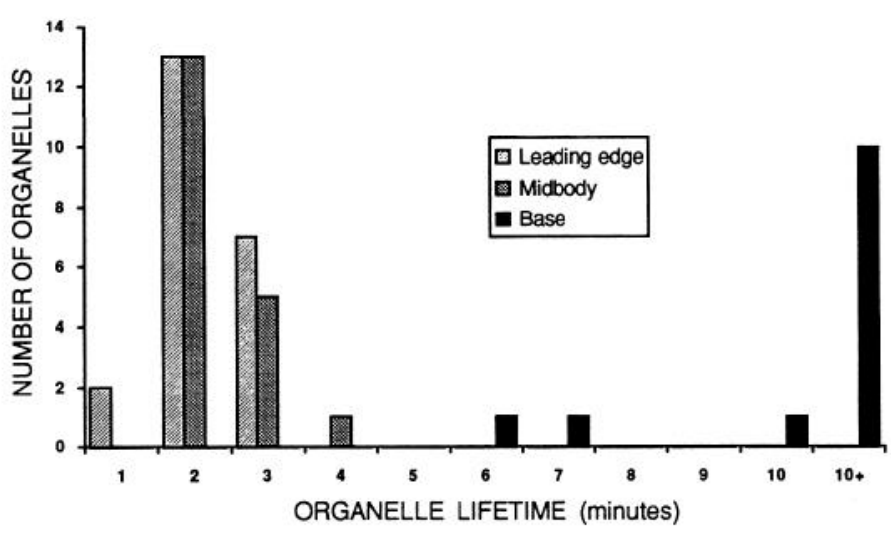

Figure 16. The lifetime of organelles with a reverse shadowcast appearance varied with site of formation. Organelles were categorized according to the location where they first became apparent in DIC video images. Organelles that first appeared near the leading edge or midbody of the growth cone invariably had a shorter lifetime than those that were formed at the growth cone base. Organelles with a relatively long lifetime were sometimes already evident at the start of the video sequence or persisted to the end of the sequence, so only a lower limit on the organelle lifetime could be determined. Therefore, organelles that were evident for longer than $10 \mathrm{~min}$ were grouped together. Some C-domain organelles were evident for at least $20 \mathrm{~min}$. attributed to differences in growth cone structure, which is related to the substrata upon which the cultures are established. In the present study, we examined cultures grown on a laminincontaining substratum, where the majority of growth cones have broad lamellipodia and relatively short filopodia (e.g., Figs. 1 and 6; see also Kleitman and Johnson, 1989). The growth cones examined by Bunge were grown on collagen and tended to be engorged with membrane organelles up to the base of filopodia, which were long and numerous. Thus, the C-domain extended virtually up to the leading edge of the growth cone, and high endocytotic activity observed there may be associated with the C-domain rather than with proximity to the leading edge of the growth cone. Alternatively, the uptake of surface membrane associated with fusion of active filopodia at the leading edge (Ludueña and Wessells, 1973; Spooner et al., 1974) may be greater for growth cones on collagen than on laminin.

\section{Membrane insertion?}

Some of the membrane interactions we observed in the EM may represent membrane addition to the growth cone surface. Although we did not see membrane organelles confluent with the plasmalemma near presumptive sites of protrusion, we did observe a number of such membrane interactions at the center of growth cones whose leading edge had the appearance of protrusion. Thus, these membrane interactions occur when at least some parts of the growth cone are expanding. Furthermore, if our interpretation of the video data is correct (see below), shrinking and disappearance of vacuoles represent fusion with and addition of membrane to the plasmalemma. These membrane interactions may last several seconds (see Fig. 14), and our freezing or fixation methods ought to preserve them. In fixed samples that had been labeled with HRP, we found two ex- 
amples of HRP-labeled vacuoles that appeared to be connected to the external space through an orifice. The simplest interpretation of this result is that these vacuoles were involved in recycling membrane: HRP was taken up during the $15 \mathrm{~min}$ incubation and the vacuoles were "caught" by fixation in the process of re-fusing with the plasma membrane and releasing the previously internalized HRP. The alternative explanation that these events represent uptake of HRP by endocytosis seems unlikely because a $3 \mathrm{~min}$ washout of HRP preceded fixation. This was more than enough time to remove all HRP from the plasma membrane; no reaction product was detected on the growth cone surface. In addition, we know from the ferritin labeling data (Table 1) that the uptake of label into vacuoles can occur within a time as short as 1-2 min. This argues against a slow uptake process requiring many minutes.

On the other hand, there are several reasons why we may not be able to detect membrane addition events in our EM specimens with equal efficiency to membrane uptake. First, protrusion and membrane expansion could have ceased during the brief (about $15 \mathrm{sec}$ ) period of specimen preparation before freezing or fixation. This seems unlikely because many growth cone regions had morphological characteristics expected of active protrusion. From our video observations we know that neurites with small growth cones having broad lamellipodia and few filopodia are almost always rapidly extending (M. E. Dailey and P. C. Bridgman, unpublished observations; see also Kleitman and Johnson, 1989), and our rapid-frozen specimens contained many growth cones with this morphology (e.g., Fig. 1). Second, it may be that most organelles that add membrane are very small compared to the vacuoles we observed interacting with the plasmalemma. Organelle-surface membrane interactions were detected on the basis of the distincive orifices in the membrane, and small orifices ( $<80 \mathrm{~nm}$ ) might be difficult to detect in the whole-mount preparation. The smallest organelle with an orifice that we detected was $150 \mathrm{~nm}$ in diameter, and the orifice had a diameter of about $100 \mathrm{~nm}$. If membrane is added by exocytosis of small vesicles, these events may be over in less than 2 msec (Heuser et al., 1979), which would decrease the probability of capturing these events even with the freezing methods used here. Nevertheless, intracellular membrane organelles were virtually never observed in regions expected to be actively protruding, which is consistent with other work (Tosney and Wessells, 1983) suggesting that local membrane addition by vesicular fusion is not necessary for protrusion. Third, it is possible that membrane addition events for net surface expansion do not occur at the growth cone.

\section{Reverse shadowcast organelles correspond to individual membrane vacuoles}

We suggest that the reverse shadowcast organelles seen in the DIC video sequences correspond to a subset of membrane vacuoles $(>150 \mathrm{~nm}$ ) observed in the EM. Their apparent size, distribution, and behavior in the video microscope are consistent with their being membrane-bound vacuoles, and the EM confirmed that membrane vacuoles have a similar distribution (i.e., at ruffles near the leading edge and within the C-domain at the base). Moreover, individual reverse shadowcast organelles could be positively reidentified in the EM as vacuoles.

In appearance and behavior, the reverse shadowcast organelles are quite similar to the large, phase-bright vacuole-like structures observed by others (e.g., Matsumoto, 1920; Lewis, 1931, 1950; Hughes, 1953; Nakai, 1956; Pomerat et al., 1967).
Pfenninger (1979) briefly discussed (but provided no data on) the behavior of these highly refractile, vacuole-like structures, stating that they "tend to increase slowly in size for several minutes and may then be seen to collapse or disappear within seconds. Over longer periods of observation, they may also change their location within the growth cone" (Pfenninger, 1979). It seems likely that the "highly refractile structures" seen by Pfenninger and others correspond to at least a subset of the reverse shadowcast organelles we observed in the DIC images. However, an investigation of the ultrastructural correlate of the highly refractile structures produced a curious result. Using thinsection EM of aldehyde-fixed growth cones, it was concluded that individual highly refractile structures consisted of clusters of large, clear vesicles (Pfenninger, 1979). Whether or not these clusters of subplasmalemmal vesicles (SPVs) are derived from the plasma membrane is uncertain because labeling experiments with ferritin have produced conflicting results (Birks et al., 1972; Wessells et al., 1976; Bunge, 1977; Pfenninger, 1979). In fact, whether the SPVs represent physiological structures or artifact is highly controversial (e.g., Hay and Hasty, 1979; Pfenninger, 1979; Rees and Reese, 1981). Our correlated LM and EM images (Figs. 7-10) revealed a one-to-one correspondence of reverse shadowcast organelles in the LM with single vacuoles in the EM. This raises the possibility that SPV clusters may sometimes be artificially derived from vacuoles during chemical fixation.

\section{Analysis of the video data: dynamics of vacuoles}

Our interpretation is that emergence of a reverse shadowcast object in the video data presented here represents formation of a vacuole-like organelle. Vacuoles may be formed by one of three different growth cone activities. First, ruffling activity may form vacuoles by trapping membrane and extracellular fluid as flailing filopodia and lamellipodia fall back onto the body of the growth cone (Hughes, 1953; Ludueña and Wessells, 1973; Spooner et al., 1974). Second, pinocytotic vacuoles may be formed by direct invagination of the surface membrane. Third, vacuole-like structures may arise by fusion of small intracellular vesicles that are not resolved in the video microscope. Although both membrane trapping and direct invagination involve internalization of surface membrane and uptake of extracellular fluid (pinocytosis), the mechanisms by which this is accomplished are somewhat different. On the one hand, invagination of the surface membrane occurs when membrane is drawn in through an inward dimple in the plane of the surface membrane. In contrast, membrane is trapped when protrusions of the surface membrane fall back onto the cell surface, and the surface membrane is reorganized to accommodate the new membrane. We propose that vacuoles are formed in the P-domain only by surface membrane ruffling, whereas $C$-domain vacuoles could be formed by invagination of the surface membrane or by fusion of existing intracellular organelles. Formation of vacuoles at sites of $\mathrm{P}$-domain ruffling may be a very fast event indeed, which may be why we did not see membrane confluence between the vacuoles and the growth cone surface. On the other hand, vacuole formation by surface membrane invagination may be slower (seconds), allowing us to capture these events.

The growth of vacuoles could be explained in one of two ways. One possibility is that growing reverse shadowcast organelles may represent invaginated pockets of the surface membrane that increase in size as more surface membrane is taken in and the pocket becomes correspondingly larger. If this is the case, then the site of invagination of the surface membrane must 
be mobile within the plane of the plasmalemma since the growing organelles sometimes moved a net distance of many times the apparent diameter of the organelle. An alternative interpretation, and one that we favor, is that organelles below the limit of resolution of the video microscope fuse with the visible organelles, causing expansion of the vacuoles. Indeed, we did observe what looked like fusion of organelles in some video sequences (e.g., Fig. 13). Although we cannot exclude the possibility that these organelles simply became too close to resolve, this seems unlikely because these same reverse shadowcast objects continued to move, shrink, and disappear normally (i.e., in a unitary manner). We did not observe stepwise shrinking as one might expect if a multicomponent object was degrading piecemeal.

Rapid diminution of the vacuoles could indicate (1) fusion of the vacuole with the plasmalemma; (2) vesiculation to small, unresolved organelles; (3) flattening of the vacuole to a lumenless membrane disk (Cheng and Reese, 1987); or (4) fusion with another intracellular membrane compartment, such as the endoplasmic reticulum (ER). Since some of the vacuoles shrank and disappeared in the P-domain, where ER is scarce (Dailey and Bridgman, 1989, 1991), we do not favor the last possibility. Also, we did not find vacuoles with an appearance suggesting that they were lumenless membrane disks in our whole-mount EM preparations, and we have observed only a single disk-like structure by thin-section EM (P. Bridgman, unpublished observations). In fact, there was also no mention of lumenless membrane disks in a previous EM study of rapid-frozen sympathetic neurons in culture (Rees and Reese, 1981), despite claims to the contrary (Gordon-Weeks, 1988). In most cases, vacuolar diminution probably did not represent vesiculation. We were able to detect vesiculation (Fig. 15), but this was a rare observation. Thus, the most likely possibility seems to be that many of the vacuoles add directly to the plasmalemma. In fact, the presence of HRP reaction product within vacuoles that were fused with the plasma membrane at a time when HRP was not present in the culture media (i.e., during washout) (Fig. 10B) is consistent with the conclusion that diminution or disappearance of the reverse shadowcast organelles represents fusion with the plasma membrane.

Once membrane was retrieved by vacuoles, it tended to accumulate within the C-domain. Vacuoles moved centripetally from the $\mathrm{P}$-domain to the $\mathrm{C}$-domain, but the reverse was never observed. Centripetal movement of organelles in the P-domain probably is related to, and perhaps dependent upon, the movement of the actin-based cytoskeleton (Fisher et al., 1988; Forscher and Smith, 1988). The tight filamentous meshwork in the P-domain may also prevent the movement of vacuoles and other organelles into the leading-edge filopodia and lamellipodia (Goldberg and Burmeister, 1986; Goldberg et al., 1992). Thus, our results indicate that if new membrane for surface expansion is added to the growth cone plasmalemma via these vacuoles, it must be spatially confined to the C-domain.

\section{Significance of membrane traffice through vacuoles}

If our interpretation is accurate, the results would suggest that much of the surface membrane taken up at P-domain ruffles is rapidly recycled at the growth cone. Vacuoles formed in the C-domain are recycled at a slower rate and may incorporate new membrane. The differences in vacuole behavior (Table 3) may be related to the method of organelle formation, and thereby reflect different functions. Vacuoles that emerge from surface membrane ruffles are probably formed by membrane trapping and may reflect retrieval of surface membrane for redistribution. Collapse of filopodia and lamellipodia onto the growth cone body could reflect a mechanism whereby probing processes that fail to find a suitable substrate are rapidly resorbed in order to supply cytoplasmic components for construction of new processes. It would, therefore, be advantageous to turn over surface components rapidly in order to sustain emerging filopodia and lamellipodia. Similarly, vacuoles found near retracting $\mathrm{C}$-domain regions may be temporary intracellular stores of surface membrane during rapid retraction of the underlying cytoskcleton. Some of the retrieved surface membrane may not be recycled locally but may instead participate in the retrograde transport of extracellular fluid from the growth cone (e.g., Hughes, 1953; Nakai, 1956). Indeed, vacuoles throughout the growth cone serve to imbibe extracellular fluid, at least transiently. This process may be important for uptake of trophic or tropic agents, which is known to occur at or near the growth cone (Campenot, 1977; Johnson et al., 1987). Since C-domain vacuoles grew in size slightly before adding to the plasmalemma, some new membrane for surface expansion may be added via these organelles. However, our data would suggest that these vacuoles are not found in numbers large enough and do not turn over rapidly enough to account for addition of a significant amount of new membrane. Therefore, they are probably not the main route of membrane addition for expansion of the surface during axon outgrowth.

\section{References}

Allen RD, Allen NS, Travis JL (1.981) Video-enhanced contrast, differential interference contrast (AVEC-DIC) microscopy: a new method capable of analyzing microtubule-related motility in the reticulopodial network of Allogromia laticollaris. Cell Motil 1:291-302.

Birks RI, Mackey MC, Weldon PR (1972) Organelle formation from pinocytotic elements in neurites of cultured sympathetic ganglia. $J$ Neurocytol 1:311-340.

Bray D (1970) Surface movements during the growth of single explanted neurons. Proc Natl Acad Sci USA 65:905-910.

Bray D (1973) Model for membrane movements in the neuronal growth cone. Nature 244:93-96.

Bridgman PC (1992) Functional anatomy of the growth cone in relation to its role in locomotion and neurite assembly. In: The nerve growth cone (Letourneau PC, Kater SB, Macagno ER, eds), pp 3953. New York: Raven.

Bridgman PC, Dailey ME (1989) The organization of myosin and actin in rapid frozen nerve growth cones. J Cell Biol 108:95-109.

Bridgman PC, Kachar B, Reesee TS (1986) The structure of cytoplasm in directly frozen cultured cells. II. Cytoplasmic domains associated with organelle movements. J Cell Biol 102:1510-1521.

Buckley IK (1974) Subcellular motility: a correlated light and electron microscopic study using cultured cells. Tissue Cell 6:1-20.

Buckley IK, Porter KR (1967) Cytoplasmic fibrils in living cultured cells. A light and electron microscopic study. Protoplasma 64:349380.

Bunge MB (1973) Fine structure of nerve fibers and growth cones of isolated sympathetic neurons in culture. J Cell Biol 56:713-735.

Bunge MB (1977) Initial endocytosis of peroxidase or ferritin by growth cones of cultured nerve cells. J Neurocytol 6:407-439.

Campenot RB (1977) Local control of neurite development by nerve growth factor. Proc Natl Acad Sci USA 74:4516-4519.

Cheng TPO, Reese TS (1985) Polarized compartmentalization of organelles in growth cones from developing optic tectum. J Cell Biol 101:1473-1480.

Cheng TPO, Reese TS (1987) Recycling of plasmalemma in chick tectal growth cones. J Neurosci 7:1752-1759.

Dailey ME, Bridgman PC (1989) Dynamics of the endoplasmic reticulum and other membrane organelles in growth cones of cultured neurons. J Neurosci 9:1897-1909.

Dailey ME, Bridgman PC (1991) Structure and organization of mem- 
brane organelles along distal microtubule segments in growth cones. J Neurosci Res 30:242-258.

Feldman EL, Axelrod D, Schwartz M, Heacock AM, Agranoff BW (1981) Studies on the localization of newly added membrane in growing neurites. J Neurobiol 12:591-598.

Fisher GW, Conrad PA, DeBiasio RL, Taylor DL (1988) Centripetal transport of cytoplasm, actin, and the cell surface in lamellipodia of fibroblasts. Cell Motil Cytoskel 11:235-247.

Forscher P, Smith SJ (1988) Actions of cytochalasins on the organization of actin filaments and microtubules in a neuronal growth cone. J Cell Biol 107:1505-1516.

Forscher P, Kaczmarek LK, Buchanan J, Smith SJ (1987) Cyclic AMP induces changes in distribution of organelles within growth cones of Aplysia bag cell neurons. J Neurosci 7:3600-3611.

Goldberg DJ, Burmeister DW (1986) Stages in axon formation: observations of growth of Aplysia axons in culture using video-enhanced contrast-differential interference contrast microscopy. J Cell Biol 103: 1921-1931.

Goldberg DJ, Burmeister DW, Rivas RJ (1992) Video microscopic analysis of events in the growth cone underlying axon growth and the regulation of these events by substrate-bound proteins. In: The nerve growth cone (Letourneau PC, Kater SB, Macagno ER, eds), pp 7995. New York: Raven.

Gordon-Weeks PR (1988) The ultrastructure of the neuronal growth cone: new insights from subcellular fractionation and rapid freezing studies. Electron Microsc Rev 1:201-219.

Hanker JS, Yates PE, Metz CB, Rustioni A (1977) A new specific, sensitive and non-carcinogenic reagent for the demonstration of horseradish peroxidase. Histochem J 9:789-792.

Hay ED, Hasty DL (1979) Extrusion of particlc-frec membranc blisters during glutaraldehyde fixation. In: Freeze fracture: methods, artifacts, and interpretations (Rash JE, Hudson CS, eds), pp 59-66. New York: Raven.

Heuser JE, Reese TS, Dennis MJ, Jan Y, Jan L, Evans L (1979) Synaptic vesicle exocytosis captured by quick freezing and correlated with quantal transmitter release. J Cell Biol 81:275-300.

Hughes A (1953) The growth of embryonic neurites: a study on cultures of chick neural tissues. J Anat 87:150-163.

Inoue S (1981) Video image processing greatly enhances contrast, quality, and speed in polarization-based microscopy. J Cell Biol 89: 346-356.

Johnson EM Jr, Taniuchii M, Clark HB, Springer JE, Koh S, Tayrien MW, Loy R (1987) Demonstration of the retrograde transport of nerve growth factor receptor in the peripheral and central nervous system. J Neurosci 7:923-929.

Kleitman N, Johnson MI (1989) Rapid growth cone translocation on laminin supported by lamellipodial not filopodial structures. Cell Motil Cytoskel 13:288-300.

Knoll G, Verkleij AJ, Plattner H (1987) Cryofixation of dynamic processes in cells and organelles. In: Cryotechniques in biological electron microscopy (Steinbrecht RA, Zierold K, eds), pp 258-271. Berlin: Springer.
Letourneau PC (1985) Axonal growth and guidance. In: Molecular basis of neuronal development (Edelman GM, Gall WE, Cowan MW, eds), pp 269-293. New York: Wiley.

Lewis WH (1931) Pinocytosis. Johns Hopk Hosp Bull 49:17-26.

Lewis WH (1950) Motion picture of neurons and neuroglia in tissue culture. In: Genetic neurology (Weiss P, ed), pp 53-65. Chicago: University of Chicago Press.

Ludueña MA, Wessells NK (1973) Cell locomotion, nerve elongation, and microfilaments. Dev Biol 30:427-440.

Matsumoto T (1920) The granules, vacuoles and mitochondria in the sympathetic nerve-fibers cultivated in vitro. Johns Hopkins Hosp Bull 31:91-93.

Nakai J (1956) Dissociated dorsal root ganglia in tissue culture. Am J Anat 99:81-129.

Pfenninger KH (1979) Subplasmalemmal vesicle clusters: real or artifact? In; Freeze fracture: methods, artifacts, and interpretations (Rash JE, Hudson CS, eds), pp 71-80. New York: Raven.

Pfenninger KH, Bunge RP (1974) Freeze-fracturing of nerve growth cones and young fibers: a study of developing plasma membrane. J Cell Biol 63:180-196.

Pfenninger KH, Maylie-Pfenninger M-F (1981) Lectin labeling of sprouting neurons. II. Relative movement and appearance of glycoconjugates during plasmalemmal expansion. J Cell Biol 89:547-559.

Pfenninger KH, de la Houssaye BA, Frame L, Helmke S, Lockerbie RO, Lohse K, Miller V, Negre-Aminou P, Wood MR (1992) Biochemical dissection of plasmalemmal expansion at the growth cone. In: The nerve growth cone (Letourneau PC, Kater SB, Macagno ER, eds), pp 111-123. New York: Raven.

Pomerat CM, Handelman WJ, Raiborn CW Jr, Massey JF (1967) Dynamic activities of nervous tissue in vitro. In: The ncuron (Hyden H, ed), pp 119-178. New York: Elsevier.

Rees RP, Reese TS (1981) New structural features of freeze-substituted neuritic growth cones. Neuroscience 6:247-254.

Sitte H, Edelman L, Neumann K (1987) Cryofixation without pretreatment at ambient pressure. In: Cryotechniques in biological electron microscopy (Steinbrecht RA, Zierold K, eds), pp 87-113. Berlin: Springer.

Spooner BS, Ludueña MA, Wessells NK (1974) Membrane fusion in the growth cone-microspike region of embryonic nerve cells undergoing axon elongation in cell culture. Tissue Cell 6:399-409.

Tosney KW, Wessels NK (1983) Neuronal motility: the ultrastructure of veils and microspikes correlates with their motile activities. J Cell Sci 61:389-411.

Weldon PR (1975) Pinocytotic uptake and intraccllular distribution of colloidal thorium dioxide by cultured sensory neurites. J Neurocytol 4:341-356.

Wessels NK, Ludueña MA, Letourneau PC, Wrenn JT, Spooner BS (1974) Thorotrast uptake and transit in embryonic glia, heart fibroblasts and neurons in vitro. Tissue Cell 6:757-776.

Wessells NK, Nuttall RP, Wrenn JT, Johnson S (1976) Differential labeling of the cell surface of single ciliary ganglion neurons in vitro. Proc Natl Acad Sci USA 73:4100-4104. 